\title{
HUSH, a Link Between Intrinsic Immunity and HIV Latency
}

\author{
Ghina Chougui ${ }^{1,2,3}$ and Florence Margottin-Goguet ${ }^{1,2,3 *}$ \\ ${ }^{1}$ Inserm, U1016, Institut Cochin, Paris, France, ${ }^{2}$ CNRS, UMR8104, Paris, France, ${ }^{3}$ Université Paris Descartes, Sorbonne \\ Paris Cité, Paris, France
}

A prominent obstacle to HIV eradication in seropositive individuals is the viral persistence in latent reservoir cells, which constitute an HIV sanctuary out of reach of highly active antiretroviral therapies. Thus, the study of molecular mechanisms governing latency is a very active field that aims at providing solutions to face the reservoirs issue. Since the past 15 years, another major field in HIV biology focused on the discovery and study of restriction factors that shape intrinsic immunity, while engaging in a molecular battle against HIV. Some of these restrictions factors act at early stages of the virus life cycle, alike SAMHD1 antagonized by the viral protein Vpx, while others are late actors. Until recently, no such factor was identified in the nucleus and found active at the level of provirus expression, a crucial step where latency may take place. Today, two studies highlight Human Silencing Hub (HUSH) as a potential restriction factor that controls viral expression and is antagonized by Vpx. This Review discusses HUSH restriction in the

OPEN ACCESS

Edited by:

Guido Poli,

Vita-Salute San Raffaele University,

Italy

Reviewed by:

Marina Lusic,

Universität Heidelberg, Germany

Fabio Romerio,

University of Maryland, United States

*Correspondence:

Florence Margottin-Goguet florence.margottin-goguet@inserm.fr

Specialty section:

This article was submitted to

Virology,

a section of the journal

Frontiers in Microbiology

Received: 16 November 2018

Accepted: 28 January 2019

Published: 12 February 2019

Citation:

Chougui $G$ and

Margottin-Goguet F (2019) HUSH, a

Link Between Intrinsic Immunity and

HIV Latency. Front. Microbiol. 10:224.

doi: 10.3389/fmicb.2019.00224 light of the actual knowledge of intrinsic immunity and HIV latency.

Keywords: restriction factors, latency, reservoirs, HIV auxiliary proteins, epigenetic control, HUSH, Vpx, Vpr

Human Immunodeficiency Virus is responsible for the Acquired Immunodeficiency Syndrome. Since its start in the early 1980s, HIV pandemic claimed about 35 million lives. In 2017, an estimated 36.9 million people were living with HIV worldwide. Despite a global decline, thanks to the increased and early access to antiretroviral therapy, in 2017, 1.8 million people were newly infected and almost 1 million died of AIDS-related illnesses (Global HIV and AIDS Statistics, 2018 fact sheet). Though many battles have been won, as HIV is no longer a death sentence but a manageable chronic illness in several parts of the world, the war for HIV eradication remains to win. Statistics above mirror the crucial need for further understanding of HIV biology and its interaction with the host, to anticipate future challenges in HIV infection control and eradication.

Lately, one major issue of HIV eradication was raised by HIV latency and precisely the persistence of reservoir cells in infected individuals, despite antiretroviral therapy. Indeed, these cells harboring integrated but silent viruses were found to be a source for viral rebound following treatment interruption. Another major field of interest is the study of restriction factors that counteract the virus at different step of its life cycle and shape intrinsic immunity. Today the characterization of the Human Silencing Hub (HUSH) complex has created a link between these two domains of interest. Here, we discuss current knowledge about restriction factors, molecular mechanisms governing HIV latency, and finally strategies to address latency. The common thread of these three parts will be a focus on HUSH.

\section{HUSH, A MOLECULAR PLAYER OF INTRINSIC IMMUNITY?}

HIV life cycle can be separated in two phases: early and late (Figure 1). The early phase extends from viral adhesion to integration, whereas late stages start from transcription to budding and 
release of the new particle (Figure 1). Throughout the whole replication process, HIV faces several barriers set up by the host cell to protect its integrity. Indeed, various cellular factors are able to detect viral elements and trigger an antiviral response. It is the case of sensors like cyclic GMP-AMP synthase (cGAS) and IFN $\gamma$-Inducible protein 16 (IFI16), which can detect HIV viral cDNA released in the cytoplasm and induce a signaling cascade resulting in the induction of interferon stimulated genes (ISGs) (Gao et al., 2013; Jakobsen et al., 2013; Lahaye et al., 2013). HIV replication is inhibited by interferon (IFN) stimulation in a celltype dependent manner, the antiviral response predominantly relies on type I interferon (IFN-I), including interferon- $\alpha$ (IFN$\alpha)$. Myxovirus resistance 2 (MX2), an IFN- $\alpha$ inducible factor, contributes to the early block of HIV replication, by preventing nuclear import of the viral cDNA (Figure 1) (Goujon et al., 2013; Kane et al., 2013; Liu et al., 2013). Moreover, antiviral factors can incorporate within the viral particle, as for IFN-induced transmembrane (IFITM) proteins. IFITM 1, 2, and 3, which are also found on the viral membrane, can hinder viral entry by interfering with viral fusion (Figure 1) (Lu et al., 2011; Compton et al., 2014; Tartour et al., 2014).

\section{Restriction Factors}

However, even before the establishment of the interferon response, a group of cellular factors have the capacity to directly interrupt diverse stages of the viral replication cycle (Figure 1). These antiviral factors constitute the cellular intrinsic defense and in order to escape from their control, lentiviruses evolved ingenious tools, namely auxiliary proteins (Figures 1, 2). Though dispensable for viral replication, at least in vitro, auxiliary or accessory proteins have the ability to specifically inactivate a small group of these cellular antiviral factors, termed restriction factors. The notion of "restriction factor" was first mentioned in the 70s, when the protective effect of Friend virus susceptibility protein 1 (Fv1) against MLV was discovered (Lilly, 1970; Pincus et al., 1971; Rowe et al., 1973). Years later, primate lentiviruses were found sensitive to such antiviral factors, yet the exact definition of a restriction factor is still debated. Indeed, diverse cellular factors are able to block HIV replication as well as other viruses, a set of criteria was therefore proposed to distinguish restriction factors from resistance factors (Harris et al., 2012; Malim and Bieniasz, 2012; Doyle et al., 2015).

Generally, at least two features are shared by restriction factors: (i) cell-autonomous antiviral activity, i.e., the ability to inhibit viral replication without the need of cell to cell communication; (ii) direct interaction with the pathogen, which in turn can escape restriction either by changing the interaction surface or inducing the targeted inactivation of the restriction factor. Due to this direct interaction and as a result of hostpathogen co-evolution, most restriction factors evolved under positive selection, i.e., the spread of protein-altering mutations. Moreover, as they are tightly linked to the cellular defense mechanism, some restriction factors can be IFN-inducible. To date only five restriction factors have been identified: Apolipoprotein B mRNA-Editing enzyme, Catalytic polypeptidelike 3G (APOBEC3G) (Sheehy et al., 2002; Conticello et al., 2003; Harris et al., 2003; Mangeat et al., 2003; Zhang et al., 2003),
Tripartite motif-containing protein 5 (TRIM $5 \alpha$ ) (Stremlau et al., 2004, 2006; Black and Aiken, 2010), Bone Stromal Tumor protein 2 (BST2)/Tetherin (Neil et al., 2008; Van Damme et al., 2008), Sterile Alpha Motif domain and HD domain-containing protein 1 (SAMHD1) (Hrecka et al., 2011; Laguette et al., 2011) and Serine Incorporator 3 and 5 (Serinc3/5) (Matheson et al., 2015; Rosa et al., 2015; Usami et al., 2015).

Apolipoprotein B mRNA-editing enzyme, catalytic polypeptide-like $3 \mathrm{G}$ and SAMHD1 are both active at early stages of HIV replication (Figure 1). SAMHD1 blocks the reverse transcription step by decreasing the pool of intracellular deoxynucleoside triphosphates (dNTP) (Goldstone et al., 2011; Hrecka et al., 2011; Laguette et al., 2011; Baldauf et al., 2012; Descours et al., 2012; Lahouassa et al., 2012), whereas the cytidine deaminase APOBEC $3 \mathrm{G}$, incorporates into the viral particle and induces $\mathrm{C}$ to $\mathrm{U}$ hyper-mutations on the viral DNA during reverse transcription, hence resulting in viral genes alteration (Sheehy et al., 2002; Conticello et al., 2003; Harris et al., 2003; Mangeat et al., 2003; Zhang et al., 2003). However, thanks to its auxiliary proteins, HIV is able to evade these two restriction factors (Figure 1). Indeed, Vif prevents the packaging of APOBEC3G by inducing its polyubiquitination, resulting in its subsequent proteasomal degradation (Sheehy et al., 2002, 2003; Mariani et al., 2003; Yu et al., 2003; Mehle et al., 2004) (Figures 1, 2). On the other hand, Vpx, which is unique to HIV-2 and not encoded by HIV-1, uses the same strategy to trigger SAMHD1 proteasomal degradation (Hrecka et al., 2011; Laguette et al., 2011) (Figures 1, 2). APOBEC3G and SAMHD1 both harbor signs of positive selection as a result of direct contact with viral proteins (Sawyer et al., 2004; Laguette et al., 2012). Although both SAMHD1 and APOBEC3G are constitutively expressed in many cell types, their expression can also be induced by IFN stimulation (Lafuse et al., 1995; Li et al., 2000; Sarkis et al., 2006; Tanaka et al., 2006; Stopak et al., 2007; Riess et al., 2017).

Alike APOBEC3G and SAMHD1, Tetherin/BST2, which is active at late stages of viral replication (Figure 1), can also be induced by IFN stimulation. BST2 prevents the release of viral particles from the producer cell by retaining virions on the cell surface. HIV-1 auxiliary protein Vpu helps the virus evade BST2 by down-regulating and sequestrating it away from viral budding sites (Neil et al., 2008; Van Damme et al., 2008). As a consequence of this viral antagonism, BST2 was shown to have evolved under positive selection (Gupta et al., 2009; McNatt et al., 2009; Lim et al., 2010).

Tripartite motif-containing protein 5 is a E3-ubiquitin ligase which can also be IFN-induced (Sakuma et al., 2007; Carthagena et al., 2008). It inhibits viral replication by binding the capsid and accelerating its proteasomal degradation, thus inducing premature uncoating which prevents reverse transcription (Stremlau et al., 2004, 2006; Black and Aiken, 2010). Interestingly and in contrast to the factors described above, TRIM5 $\alpha$ is not targeted by a viral protein. Instead, retroviruses evade its restriction through capsid mutation, hence altering the interaction surface and avoiding recognition by TRIM5 $\alpha$ (Hatziioannou et al., 2006; Kamada et al., 2006). This also explains why rhesus monkey but not human TRIM5 $\alpha$ inhibits HIV replication (Stremlau et al., 2004, 2006; 


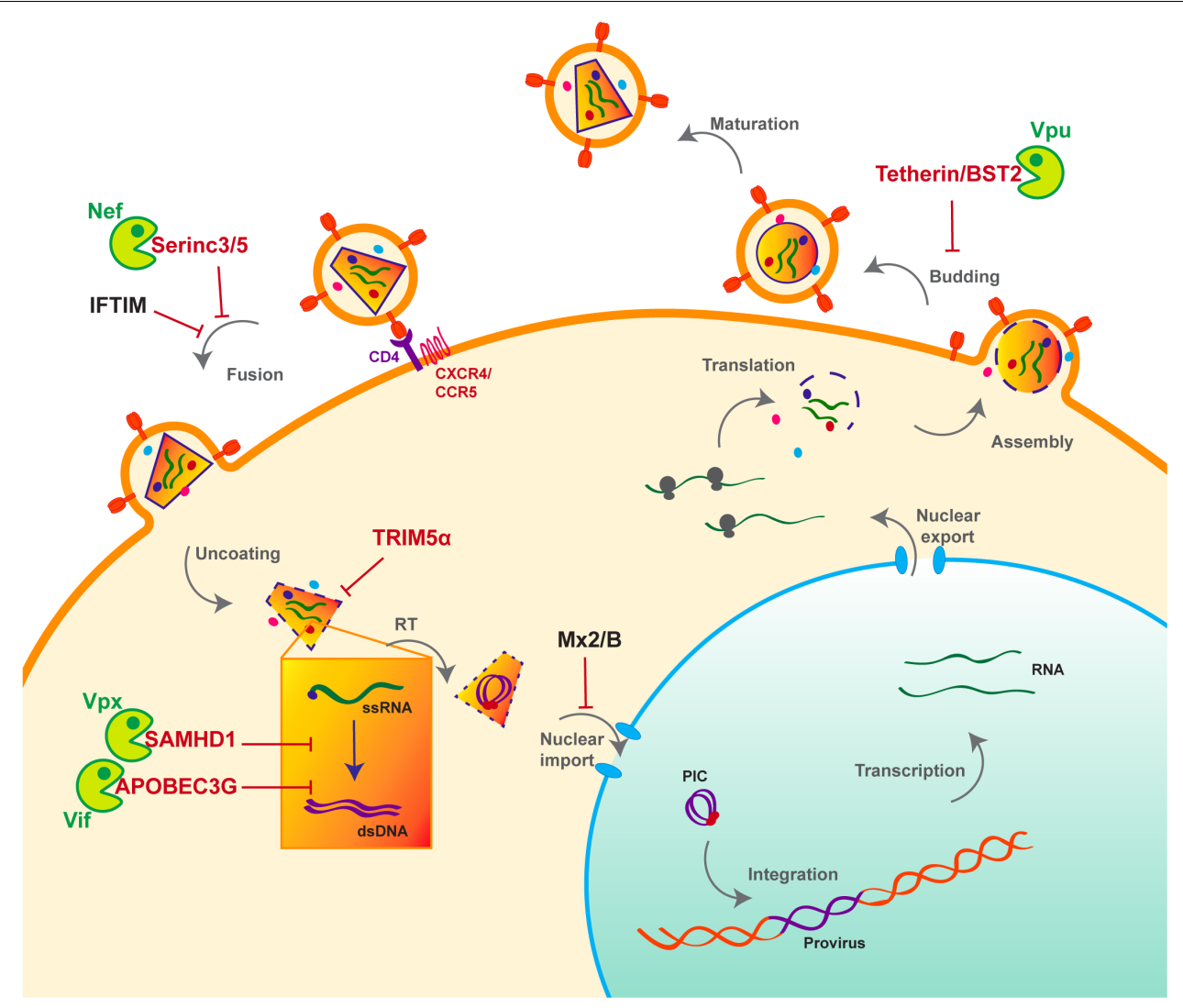

FIGURE 1 | Host antiviral factors and viral agonists. Following virion adhesion to the cellular receptor and coreceptor, HIV enters the cell through fusion of its envelope with the cellular membrane. The capsid is therefore destabilized and Reverse transcription (RT) is triggered, allowing the synthesis of a double stranded DNA (dsDNA) using the viral single stranded RNA (ssRNA) as a matrix. Viral DNA along with other viral proteins including Vpr, Matrix (MA) and Integrase (IN) form the pre-integration complex (PIC), which is imported into the nucleus thanks to Nuclear Localization Signals (NLS) (Brown et al., 1987). This nuclear import step is critical as it allows HIV to infect non-dividing cells, in contrast to other retroviruses such as HTLV, which requires nuclear membrane destabilization. After viral DNA integration, viral genes are transcribed by the cellular machinery and the viral protein Tat; various forms of spliced RNAs are exported from the nucleus for translation. Unspliced RNAs are escorted by the viral protein Rev and will constitute the viral genome of the newly assembled virion. Finally, immature viral particles bud at the membrane packaging both viral and cellular factors. Constitutively expressed antiviral restriction factors are in red, others are in black. Restriction factors might be antagonized by viral auxiliary proteins, shown in green, some being specific to HIV-1/SIVcpz (Vpu), other to HIV-2/SIVmac/SIVsmm (Vpx).

Black and Aiken, 2010). TRIM5 $\alpha$ is therefore an important barrier for cross-species transmission, which also evolved under positive selection (Sawyer et al., 2005; Tareen et al., 2009).

Based on their features and compared to the previously described factors, Serinc 3 and 5 are intriguing. Serinc 3 and 5 are both packaged into new virions and inhibit viral fusion with the target cell (Matheson et al., 2015; Rosa et al., 2015; Usami et al., 2015). HIV-1 uses Nef to evade Serinc $3 / 5$ and induces its down-regulation. Nef dramatically increases virions infectivity by preventing Serinc3/5 incorporation through its removal from cell surface (Matheson et al., 2015; Rosa et al., 2015; Usami et al., 2015). Serinc $3 / 5$ is not up-regulated by IFN and despite its interaction with Nef, no sign of molecular arm race were found in Serinc3/5, meaning no recent virus-host co-evolution (Murrell et al., 2016).

\section{The Molecular Arm Race: Adapt to Thrive}

Interaction with pathogens, including viruses, profoundly shaped the evolutionary history of human intrinsic immunity and particularly that of restriction factors, which very often are in direct contact with these threats (Duggal and Emerman, 2012). Restriction factors represent a selective pressure on viruses but once these viruses mutate to escape restriction, adapted cellular factors then provide a fitness advantage to their host. Therefore, a competition for continuous adaptation arises between the virus and its host, termed "the molecular arms race." Such genetic conflicts between host and viral proteins frequently results in events of "positive selection," meaning an excess of non-synonymous mutations $(\mathrm{dN})$ compared to synonymous mutations (dS). Positively selected sites often correspond to the interaction interface between host and viral factors, such sites are found in TRIM5 $\alpha$, APOBEC3G, Tetherin/BST2, and SAMHD1 (Sawyer et al., 2004, 2005; McNatt et al., 2009; Lim et al., 2010; Laguette et al., 2012).

Nonetheless, due to constrains related to their cellular role, positive selection is not the only strategy used by hosts' factors to cope with viral escape, other events such as polymorphism, gene duplication and various innovations including the presence 


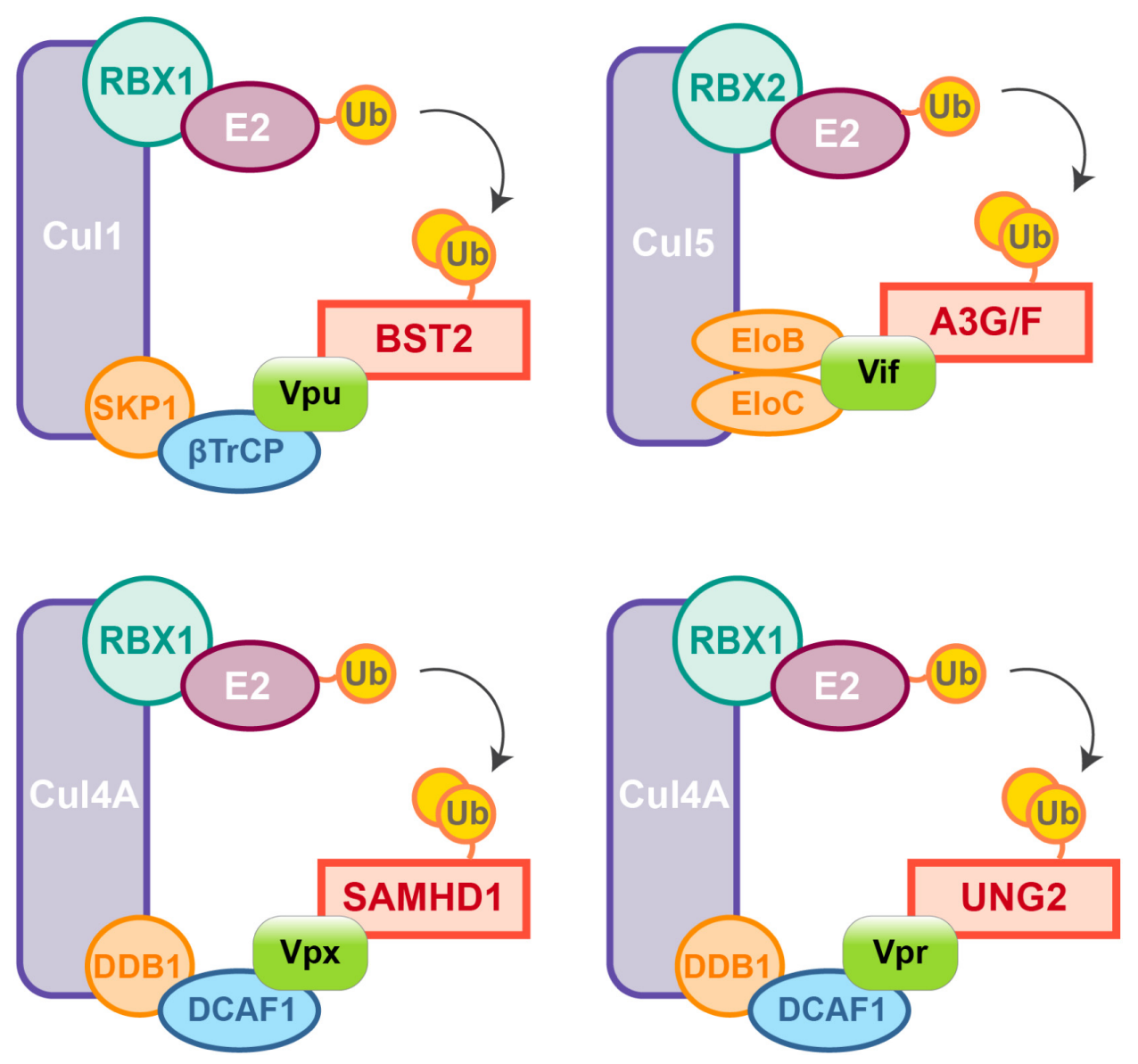

FIGURE 2 | HIV auxiliary proteins hijack the ubiquitin-proteasome pathway. HIV auxiliary proteins hijack different Cullin-RING Ligases (CRLs). CRLs share the same architecture, they are built on a Cullin scaffold (purple) binding a RING-box protein (RBX1 or 2, green) along with adaptor proteins (blue and orange) which allow substrate recognition. Vif induces APOBEC3G and F degradation by usurping the E3 ubiquitin ligase Cul5, Vif mimicks the adaptor SOCS (not represented) and directly interacts with the adaptors Elongin B and C. In contrast, HIV-1 Vpu hijacks the E3 ubiquitin ligase Cul1 through interaction with the $\beta$ TrCP adaptor. HIV-2/SIVsmm Vpx uses the same strategy and binds the adaptor DCAF1 to recruit the E3 ubiquitin ligase Cul4A and induces proteasomal degradation of SAMHD1. Cul4A-DDB1 ${ }^{\text {DCAF1 }}$ ubiquitin ligase is also hijacked by HIV-1 Vpr to target the Uracyl DNA Glycosylase (UNG2) (Ahn et al., 2010).

of different isoforms are observed, alike APOBEC3 family which counts seven paralogs with cytosine deaminase activity. Although less active against HIV than APOBEC3G, APOBEC3D, $\mathrm{F}$, and $\mathrm{H}$ show restricting activities but only APOBEC3F and one haplotype of APOBEC3H were found counteracted by Vif (Wiegand et al., 2004; Liu et al., 2005; Binka et al., 2012). On the other hand, despite lower fidelity of their reverse transcriptase resulting in higher mutation rates, viruses also evolve under tight constrains, a consequence of their small genome size and overlapping open reading frames (ORFs). Some viral proteins can also have several roles and interact with both viral and cellular factors. Furthermore, in order to antagonize a single restriction factor, in addition to direct binding of the target, a viral protein may require interaction with a secondary host factor. It is the case of viral-induced proteasomal degradation of cellular factors, which relies on the hijacking of cellular ubiquitin ligases, especially Cullin-RING Ligases (CRLs) in the case of HIV (Figure 2). For instance, Vpx requires the DDB1-Cullin4associated factor 1 (DCAF1) as an adaptor to hijack the Cul4A ubiquitin ligase (Figure 2) (Le Rouzic et al., 2007; Sharova et al., 2008; Srivastava et al., 2008).

Viral protein $\mathrm{R}$ (Vpr), a paralog of Vpx usurps the same ubiquitin ligase but does not degrade SAMHD1. Instead Vpr has different functions among them the mysterious induction of cellcycle arrest in dividing cells (He et al., 1995; Jowett et al., 1995; Re et al., 1995; Le Rouzic et al., 2007; Wen et al., 2007; Sharova et al., 2008; Srivastava et al., 2008).

\section{SAMHD1 Antagonism by Vpx}

Viral protein $\mathrm{R}$ and $\mathrm{X}(\mathrm{Vpx})$ were identified in the early 90's, they are incorporated into the viral particle and thus present at early stages of viral replication (Henderson et al., 1988; Kappes et al., 1988; Cohen et al., 1990a,b). These two proteins share the same three-helix bundle structure and are both able to bind DCAF1 through residues within the third $\alpha$-helix (Figure 3A).

$V p r$ and $v p x$ genes are thought to originate from complex events of duplication and/or recombination of one common precursor gene (Sharp et al., 1996; Tristem et al., 1998), but in 


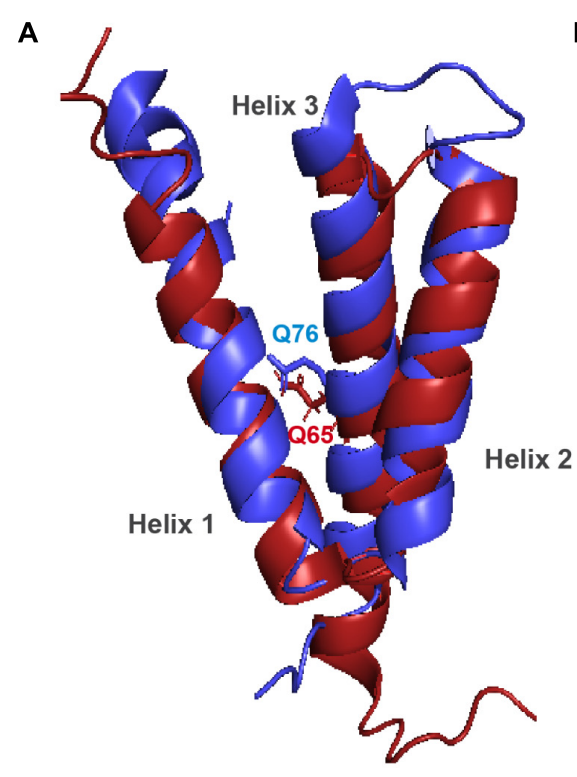

${ }^{B}$

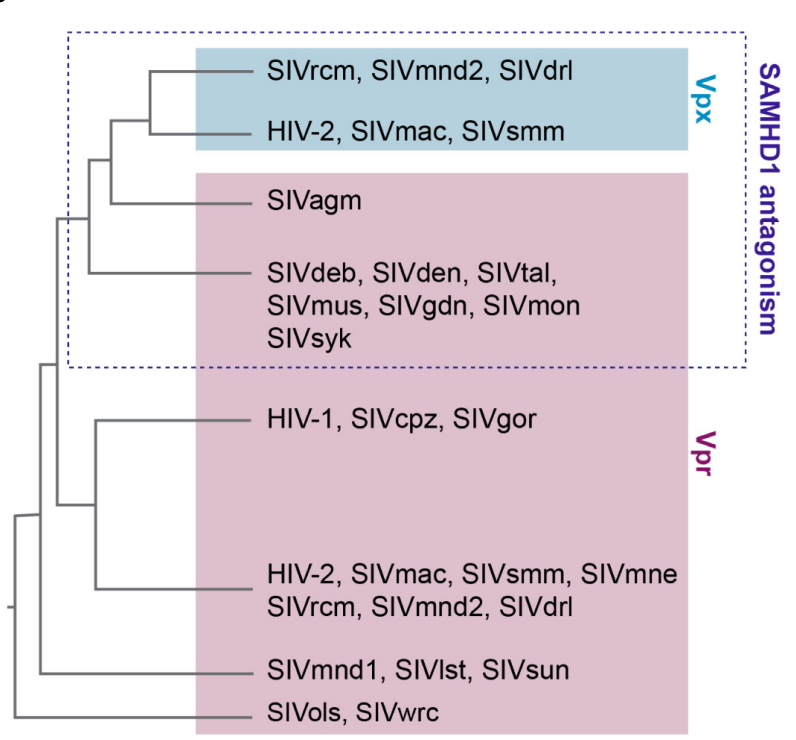

FIGURE 3 | Structure comparison and origin of Vpr and Vpx. (A) Structural similarities. Alignment of the 3D PDB structures of HIV-1 Vpr (red) and SIVsmm Vpx (blue). Three $\alpha$-helix structures are separated with loops and two flexible regions at both Nter and Cter ends. One residue critical for DCAF1 binding is represented on each structure, Q65 on HIV-1 Vpr and Q76 on SIVsmm Vpx. In contrast to Vpr, Vpx is stabilized by a zinc finger motif [PDB:1M8L (Morellet et al., 2003), SIVsmm Vpx (PDB: 4cc9 (Schwefel et al., 2014)]. (B) Schematic representation of a VpxNpr phylogenic tree. Vpx are in the blue box, Vpr are in the pink, based on (Lim et al. 2012).

contrast to $v p r$ which is found in all primate lineages, its paralog, $v p x$, is only present in two lineages: HIV-2/SIVsmm/SIVmac and SIVrcm/SIVmnd2 from red-capped monkeys and from mandrill (Figure 3B) (Beer et al., 2001; Hu et al., 2003).

Interestingly, despite divergence of their sequences, $v p x$ genes from different lineages cluster together away from their homologous $v p r$ genes from the same lineage (Figure 3B). Furthermore, though these two proteins hijack the same ubiquitin ligase, Vpr and Vpx bear different functions. Vpr has the mysterious ability to induce cell-cycle arrest in dividing cells, which contribution to viral replication remains unknown, whereas Vpx induces SAMHD1 degradation, hence relieving a block on reverse transcription. However, some $\mathrm{Vpr}$ from lineages lacking Vpx are exceptions as they possess both of these functions, alike Vpr from SIVagm and SIVsyk, respectively, from African green monkey and Syke's monkey (Stivahtis et al., 1997; Lim et al., 2012).

In contrast to the mystery surrounding the role of $\mathrm{Vpr}$, $\mathrm{Vpx}$ function during viral replication is rather well understood, at least partly. This $12-16 \mathrm{kDa}$ protein is incorporated into the viral particle and expressed by only two lineages as stated above. Although Vpx seems dispensable for viral replication in lymphocytic cell lines (Yu et al., 1988; Hu et al., 1989), in vivo its deletion was reported to negatively impact SIV spread and kinetics in monkeys (SIVsmm, SIVmac, and SIVmne from pig-tailed macaques) (Gibbs et al., 1995; Hirsch et al., 1998; Belshan et al., 2012). Loss of Vpx was also shown to drastically impair viral replication at early stages in activated peripheral blood mononuclear cells (PBMCs), primary lymphocytes and, with even greater effects in monocyte-derived macrophages
(MDMs) (Guyader et al., 1989; Kappes et al., 1991; Yu et al., 1991; Gibbs et al., 1994; Kawamura et al., 1994; Park and Sodroski, 1995). Moreover, viral transduction with both SIVmac and HIV-1-derived lentivectors was increased following Vpx delivery through virus-like particle (VLP) in MDMs and in monocyte-derived dendritic cells (MDDCs) (Goujon et al., 2006), such effect was, however, absent in activated primary $\mathrm{T}$ cells. The same positive impact of Vpx was further observed on HIV-2/SIVsmm and HIV-1 full length viruses and was shown to depend on the proteasome, precisely on the hijacking of the Cul4A-DDB1 ${ }^{\text {DCAF1 }}$ ubiquitin ligase (Goujon et al., 2007; Fujita et al., 2008a; Sharova et al., 2008; Srivastava et al., 2008; Bergamaschi et al., 2009). Vpx activity was found critical for the reverse transcription step in MDMs, in which the lack of Vpx strongly reduced viral DNA synthesis, a phenomenon observed with Feline Immunodeficiency virus (FIV) and MLV as well (Goujon et al., 2007; Fujita et al., 2008a; Sharova et al., 2008; Srivastava et al., 2008; Bergamaschi et al., 2009). Altogether, these observations demonstrated the existence of an early block on viral replication in myeloid cells, which was not specific to HIV-2/SIVsmm viruses, but counteracted by Vpx through ubiquitination. Vpx was therefore expected to inactivate, via the proteasome, a restriction factor active at reverse transcription and specific of myeloid cells. This model was finally confirmed after the identification of the Vpx target SAMHD1 (Hrecka et al., 2011; Laguette et al., 2011), which was later found also active in quiescent T cells (Baldauf et al., 2012; Descours et al., 2012).

Several Vpx mutants have been described as reviewed in Schaller et al. (2014). Vpx Q76A or Q76R and K77A, which no longer bind DCAF1, were found both unable to induce 
SAMHD1 degradation (Srivastava et al., 2008; Bergamaschi et al., 2009; Hrecka et al., 2011). Wild-type Vpx and the Vpx Q76A mutant were shown to rescue HIV-1 infection in IFN-treated MDDCs (Pertel et al., 2011), independently from dNTP levels and after completion of reverse transcription (Reinhard et al., 2014). These results suggested the existence of another Vpx strategy to antagonize SAMHD1 or a second IFN-inducible target of Vpx. In addition, Vpx deletion was reported to impair viral replication in activated PBMCs and lymphocytes (Guyader et al., 1989; Kappes et al., 1991; Yu et al., 1991; Akari et al., 1992), in which SAMHD1 is thought to be inactive. Such replication defect due to Vpx deletion was also observed in HSC-F cells that express undetectable levels of SAMHD1 (Ueno et al., 2003; Fujita et al., 2008b). Finally, Vpx from SIVmnd2/SIVrcm were recently reported to counteract a SAMHD1-independent restriction in human resting CD4+ T cells (Baldauf et al., 2017). Taken together, these observations indicate the existence of additional Vpx targets, unrelated to SAMHD1.

\section{HUSH Antagonism by Vpx and Vpr Lentiviral Proteins}

Two recent studies, including ours, identified HUSH as a cellular complex inactivated by Vpx (Chougui et al., 2018; Yurkovetskiy et al., 2018). The TASOR/FAM208A component of HUSH appeared first as c3orf63 in an siRNA screen for HIV antiviral factors (Liu et al., 2011), then in a screen for indirect chromatin reader in mouse tissues (Eberl et al., 2013). Also, in a study aimed at identifying factors involved in the epigenetic control, Harten et al. (2014) mutagenized a mouse cell line carrying a multicopy transgene expressing GFP under the control of the human alpha globin promoter. In this mouse line, $45 \%$ of erythrocytes do not express GFP as a result of epigenetic silencing, thus Harten et al. (2014) selected mutants with an increased percentage of GFP expressing cells and generated mutant mouse lines, among which MommeD6 and MommeD20, the first mice heterozygous mutants for FAM208A. Of note, in this model, FAM208A was also found to be critical for normal mouse development (Harten et al., 2014). Then, FAM208A was renamed as TASOR for Transgene Activation SupreressOR, a member of an epigenetic complex involved in chromatin silencing, the HUSH complex (Tchasovnikarova et al., 2015). In this study, the HUSH complex was identified in a screen for factors involved in position-effect variegation (PEV), i.e., the silencing of a normally active gene as a result of its positioning into heterochromatin (Elgin and Reuter, 2013). Namely, Tchasovnikarova et al. (2015) transduced the near-haploid cell line KBM7 with a lentiviral construct expressing GFP from the spleen focus-forming virus (SFFV) promoter and then sorted the population of transduced cells with a low GFP expression (GFP dim). Following mutagenesis of the GFPdim population and the analysis of mutant cells with an increased GFP expression (GFP ${ }^{\text {bright }}$ ), SETDB1, Family protein with sequence similarity 208A (FAM208A), Matrix Metalloproteinase-8 (MPP8) and Periphilin appeared as critical repressors of transgene expression. Tchasovnikarova et al. (2015) study found FAM208A, renamed as TASOR, to interact with both Periphilin and MPP8, forming the HUSH complex, which

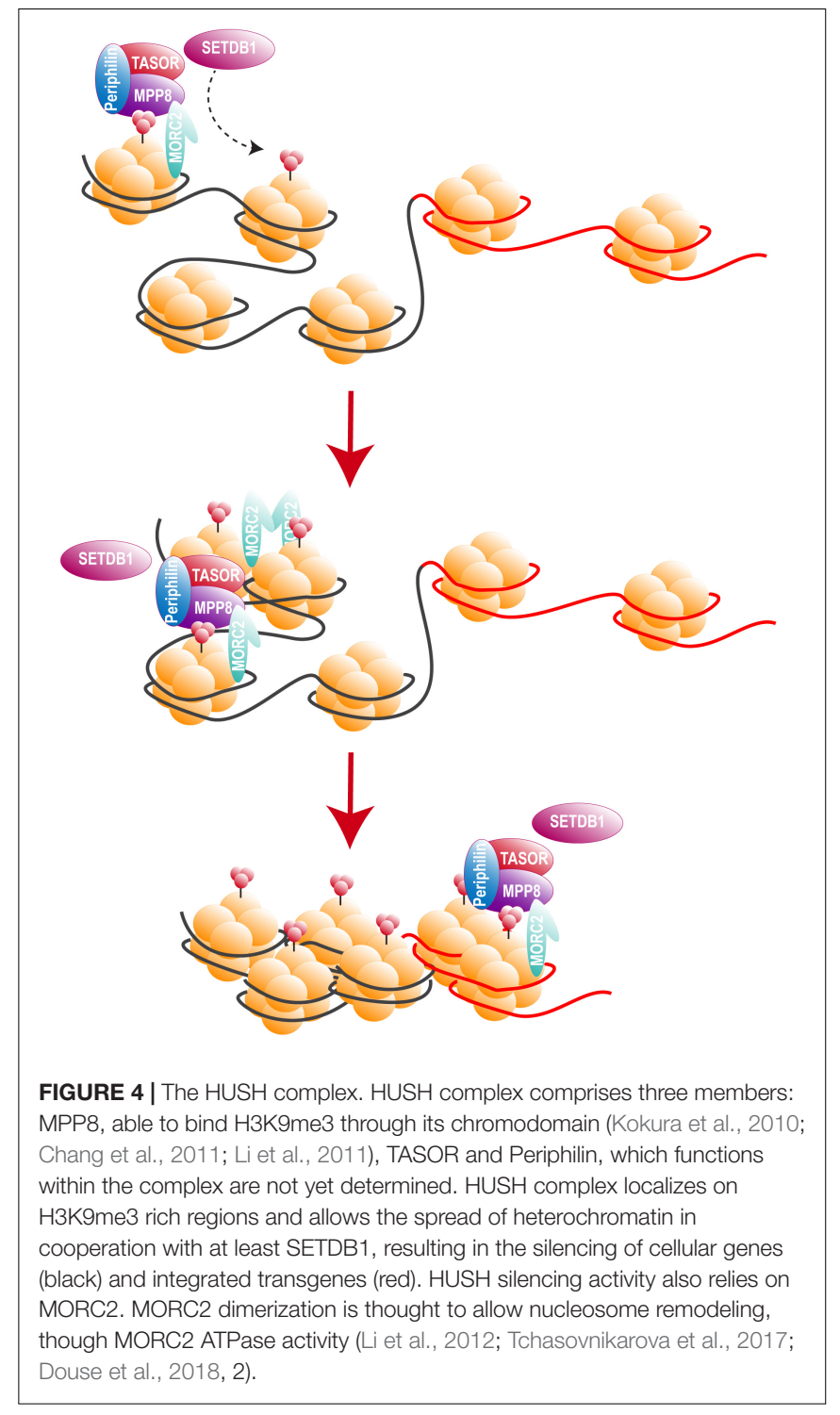

in turn recruited the methyl transferase SETDB1 (Figure 4). It was therefore suggested that HUSH complex could maintain transcriptional silencing by facilitating the spread of H3K9me3 marks on integrated transgenes (Figure 4).

In our study, we retrieved TASOR as the second best downregulated target of Vpx, just after SAMHD1, by performing a Stable-Isotope Labeling by Amino acids in Cell culture (SILAC) proteomic screen (Chougui et al., 2018). Tasor was also selected in a loss-of-function Vpx study focused on genes that contribute to silencing of retroviruses (Yurkovetskiy et al., 2018). Both studies further showed that Vpx interacts with HUSH and induces its proteasomal degradation through the hijacking of DCAF1, using the same mechanism as the one described for SAMHD1 antagonism. Though, HUSH antagonism by Vpx is genetically separable from SAMHD1 antagonism as revealed by a Vpx mutant defective for HUSH degradation, but still able to inactivate SAMHD1 (Chougui et al., 2018). Mimicking 


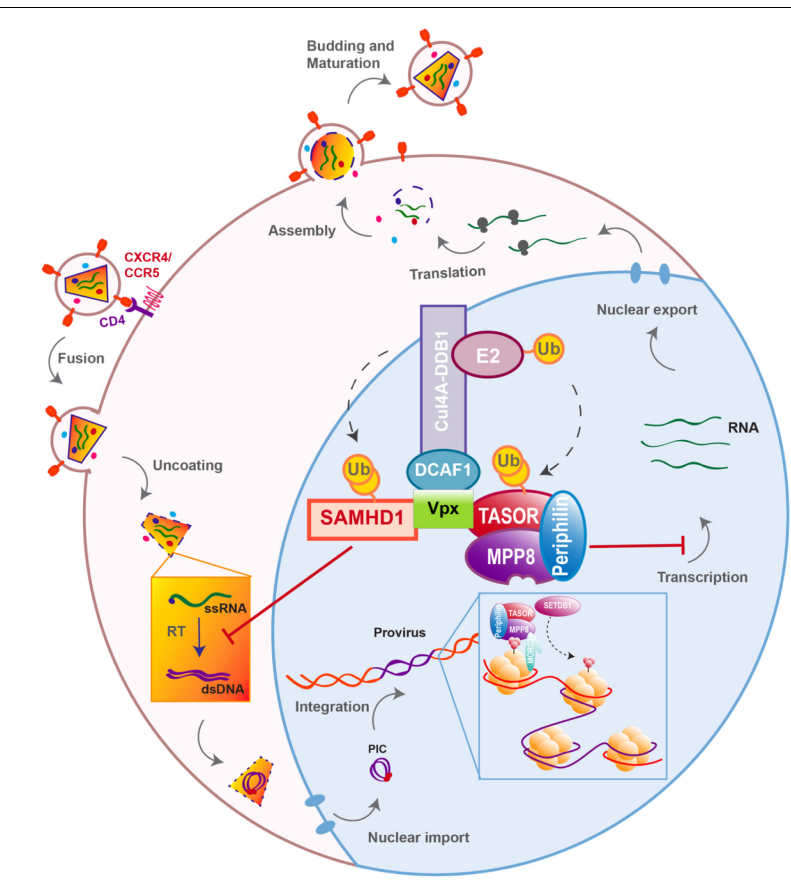

FIGURE 5 | Vpx overcomes two independent blocks on viral replication. To simplify the scheme, ubiquitination and binding of the HUSH complex is represented through TASOR.

HUSH depletion, Vpx induces the reactivation of a latent HIV1 provirus through $\mathrm{H} 3 \mathrm{~K} 9 \mathrm{me} 3$ marks depletion in the J-Lat A1 model of latency (Chougui et al., 2018). Furthermore, HUSH depletion gives an advantage to replication-competent HIV1 or SIVmac239- $\Delta \mathrm{Vpx}$ viruses (Yurkovetskiy et al., 2018). Altogether, these data report a new role of Vpx that counteracts HUSH, a complex active at the post-integration level (Figure 5). Interestingly, this unexpected function is both independent from SAMHD1 and unrelated to the previously described SAMHD1independent effects of Vpx, either in IFN-treated MDDCs (Pertel et al., 2011; Reinhard et al., 2014) or in quiescent CD4+ T cells, which seems to take place early at the level of reverse transcription (Baldauf et al., 2017). These observations therefore suggest the existence of remaining Vpx targets besides SAMHD1 and the HUSH complex.

\section{Is HUSH a Restriction Factor?}

Alike SAMHD1, HUSH complex is constitutively active in a cellautonomous manner and is counteracted by at least one viral protein through proteasomal degradation, via the hijacking of a cellular E3 ubiquitin ligase. However, global evolutionary analysis failed to identify residues under positive-selection, as it is the case with Serinc3/5. Instead, members of the HUSH complex exist in multiple and variable isoforms as it is the case for TASOR and Periphilin (which has also undergone HERV-M insertion), such diversifications can be considered as signatures of a potential gene-conflict (Huh et al., 2006; Chougui et al., 2018).

Antagonism of HUSH complex by $v p x / v p r$ genes is ancestral and precedes the birth of Vpx and SAMHD1 antagonism. It is also a species-specific function as some but not all $\mathrm{Vpr} / \mathrm{Vpx}$ were able to recognize the human HUSH complex. However, testing for human HUSH recognition gives no indication on whether these inactive $\mathrm{Vpr} / \mathrm{Vpx}$ maintain or not this function against HUSH complexes from their corresponding species. Indeed, although Vpx from SIVrcm-GAB1 did not degrade human HUSH complex as found in our study, Vpx from other SIVrcm strains did (NG411 and 02CM8081), as shown in Yurkovetskiy et al. (2018). In fact, we already have observed such differences in the capacity to recognize and degrade human HUSH complex when testing different Vpr from SIVagm. Here again, while Vpr from SIVagm.ver-KE.9063 and SIVagm.sab-1 degraded human HUSH, SIVagm.gri-677 did not, these results were also reproduced and extended to other SIVagm Vpr in Yurkovetskiy et al. (2018). Unfortunately, both Yurkovetskiy et al. (2018) and our study lack positive controls for the functionality of Vpr/Vpx that do not degrade human HUSH.

Overall, although the capacity of these $\mathrm{Vpr} / \mathrm{Vpx}$ to recognize the HUSH complex of their corresponding species were not yet investigated, these results encourage the idea of a molecular arms race with perhaps limited possibilities of evolution for the members of the HUSH complex. Strong constrains on HUSH members may have arisen from the crucial cellular role played by this complex in maintaining genomic integrity and regulating the expression of more than 900 cellular genes (Tchasovnikarova et al., 2015). HUSH complex activity also have critical implications in developmental process as mice mutant for TASOR were found not viable beyond gastrulation (Harten et al., 2014; Bhargava et al., 2017). Moreover, besides the need to maintain interactions within the complex, cooperation with both MORC2 and SETDB1 were found required for the functioning of the HUSH complex (Tchasovnikarova et al., 2015, $2017,2)$. Adding to this, HUSH complex and especially TASOR was found to collaborate with Tripartite motif-containing 28 (TRIM28) in the silencing of L1 elements (Robbez-Masson et al., 2018). The existence of numerous partners may have further reduced the possible evolutionary trajectories for members of the HUSH complex, thus the identification of critical sites under positive-selection may require beforehand isolation and analysis of precise regions within the complex that are at the interface with the viral proteins $\mathrm{Vpx} / \mathrm{Vpr}$. Of note, direct interaction between $\mathrm{Vpx}$ and members of HUSH has not been investigated yet, it is not excluded that cellular intermediates bridge the viral protein to the epigenetic repressor complex.

Finally, in the light of our actual knowledge, HUSH complex seems to share common features with restriction factors, especially with Serinc 3/5. Future studies will have to determine whether HUSH complex is wired to the immune system, by assessing the impact of IFN stimulation on its expression and antiviral activity.

\section{HIV-2 Clinical Features: Is HUSH Antagonism by Vpx a Paradox?}

In contrast to HIV-1, HIV-2 is less infectious with lower rates of transmission and is restricted to West Africa. HIV-2 is 
less pathogenic with low plasma viremia and $86-95 \%$ of HIV2 infected patients are considered long-term-non-progressors, whereas only 5-15\% HIV-1 positive patients are (MartinezSteele et al., 2007). Overall, HIV-2 displays a higher tendency for viral latency as reviewed in Saleh et al. (2017), thus Vpx capacity to counteract HUSH raises questions regarding the clinical outcomes of HIV-2. Indeed, one would expect HUSH antagonism by $\mathrm{Vpx}$ to result in a highly expressed HIV-2 instead of the weak expression observed in patients. However, HIV-2 is also able to counteract SAMHD1, APOBEC3G, and Tetherin/BST2, yet it remains a low expressed virus. Therefore, clinical features does not seem to necessarily correlate with the restriction factors a virus can overcome, as also demonstrated in a study which found no differences in SAMHD1 antagonism by $\mathrm{Vpx}$ alleles from HIV-2 viremic and long-term aviremic patients (Yu et al., 2013).

HIV-2 low expression may result from a stronger immunological control, as HIV-2 triggers a greater immuneresponse in patients than HIV-1 does, reviewed in Nyamweya et al. (2013). One could imagine the targeting and degradation of too many restriction factors by $\mathrm{HIV}-2$ to be responsible for a better immune sensing. Being too sensitive to these restriction factors, HIV-2 may have evolved tools to escape their repression but with a considerable cost on its fitness i.e., an increased vulnerability to detection by the immune system.

However, such hypothesis is less likely as SIVsmm, at the origin of $\mathrm{HIV}-2$, replicates intensively in its natural hosts without inducing AIDS and shows high levels of plasma viremia, which can be even higher than those observed in HIV-1 patients (Rey-Cuillé et al., 1998; Silvestri et al., 2003). Furthermore, SIVsmm infection is characterized by lower levels of immune activation than observed with HIV2 (Silvestri et al., 2003, 2005; Sumpter et al., 2007). The immunological control of HIV-2 might therefore arise from rather a lack of adaptation of this virus to its new host, nonetheless, HIV-2 viral expression is still high enough to allow viral transmission.

\section{Impact of HUSH Antagonism on the Epigenetic Landscape}

Overall, HUSH antagonism by Vpx raises questions over the impact of HIV-2 infection on the epigenetic landscape and subsequently on cellular genes as well as exogenous element expression. If HUSH degradation is confirmed in cells from infected patients, then it would be interesting to asses expression of genes under the control of the HUSH complex and to further evaluate its possible consequences on HIV infection and in terms of disease development. Indeed, HUSH complex is involved in the epigenetic control of more than 900 loci, with many genes belonging to the Krüppel-associated box (KRAB) domain-containing zinc-finger proteins (KZFPs) family of transcription repressors (Tchasovnikarova et al., 2015). KZFP proteins are involved in various process ranging from development to metabolism and even cancer as reviewed in Lupo et al. (2013).

\section{WHAT POTENTIAL ROLE FOR HUSH IN THE CONTEXT OF HIV LATENCY?}

Activated CD4+ T cells are highly susceptible to HIV infection, in most cases viral replication results in the death of the infected cell. However, some infected $\mathrm{T}$ cells survive long enough to return to a resting state, as memory $\mathrm{T}$ cells. This reversible shift from activated to resting state is a normal physiological process, allowing rapid immune responses following re-exposure to an antigen. As resting state is non-permissive for viral replication, these long-lived memory $\mathrm{T}$ cells will bear a silent but replication-competent provirus, therefore constituting the major latent reservoir (Chun et al., 1997; Finzi et al., 1999; Chomont et al., 2009). These reservoirs are established early after infection, initiation of cART during primary infection was shown to reduce the size of these reservoirs and allow a better control of viremia following treatment interruption (Chun et al., 1998; Strain et al., 2005; Archin et al., 2012b; Buzon et al., 2014). Nonetheless, even under prolonged antiretroviral therapy, latent reservoir cells persist (Siliciano et al., 2003), forming an HIV sanctuary out of reach of conventional therapies.

\section{Molecular Mechanisms of Latency}

Latency is a complex, multifactorial process that involves different cells, cellular subsets and the combination of several molecular mechanisms (Margolis, 2010), only three points will be discussed below: availability of cellular factors, integration site and chromatin-mediated repression.

Two identical Long Terminal Repeat (LTR) sequences are located at both extremities of HIV provirus (Figure 6). U3 region of the $5^{\prime}$ LTR serves as a promoter recruiting cellular transcription factors and the RNA pol II. Due to the elongation block of the RNA pol II by negative factors (Wu et al., 2003), incomplete viral transcripts will be synthesized, these transcripts will, however, allow the production and accumulation of the viral trans-activator protein (Tat). Tat is necessary for elongation activation (Kao et al., 1987), it binds the trans-activation response element (TAR) (Figure 6), and subsequently recruits the positive transcription elongation factor-b (P-TEFb). This will result in a positive feedback loop and only then will the HIV transcription be efficiently activated. Thus, the triggering of HIV transcription critically relies on the host's factors availability, some of which are inducible and dependent on the cellular state, as for NF-кB and NFAT that are found sequestrated in the cytoplasm in the absence of cellular activation signals. Stimulation of latently infected cells with phorbol myristate acetate (PMA), phytohemagglutinin (PHA) or Tumor Necrosis Factor $\alpha(\mathrm{TNF} \alpha)$, results in the reactivation of HIV transcription in several latency model cells. Low levels of transcription factors may therefore contribute to the non-permissive state of resting CD4 $+\mathrm{T}$ cells, along with $\mathrm{P}-\mathrm{TEFb}$ levels that show a drastic increase during cellular transition from quiescent to activated state (Budhiraja et al., 2013).

Due to integrase interaction with Lens epithelium-derived growth factor (LEDGF/p75) (Llano et al., 2006; Shun et al., 2007), HIV-1 favors integration into introns of actively transcribed genes, near the nuclear pores (Schröder et al., 2002; 


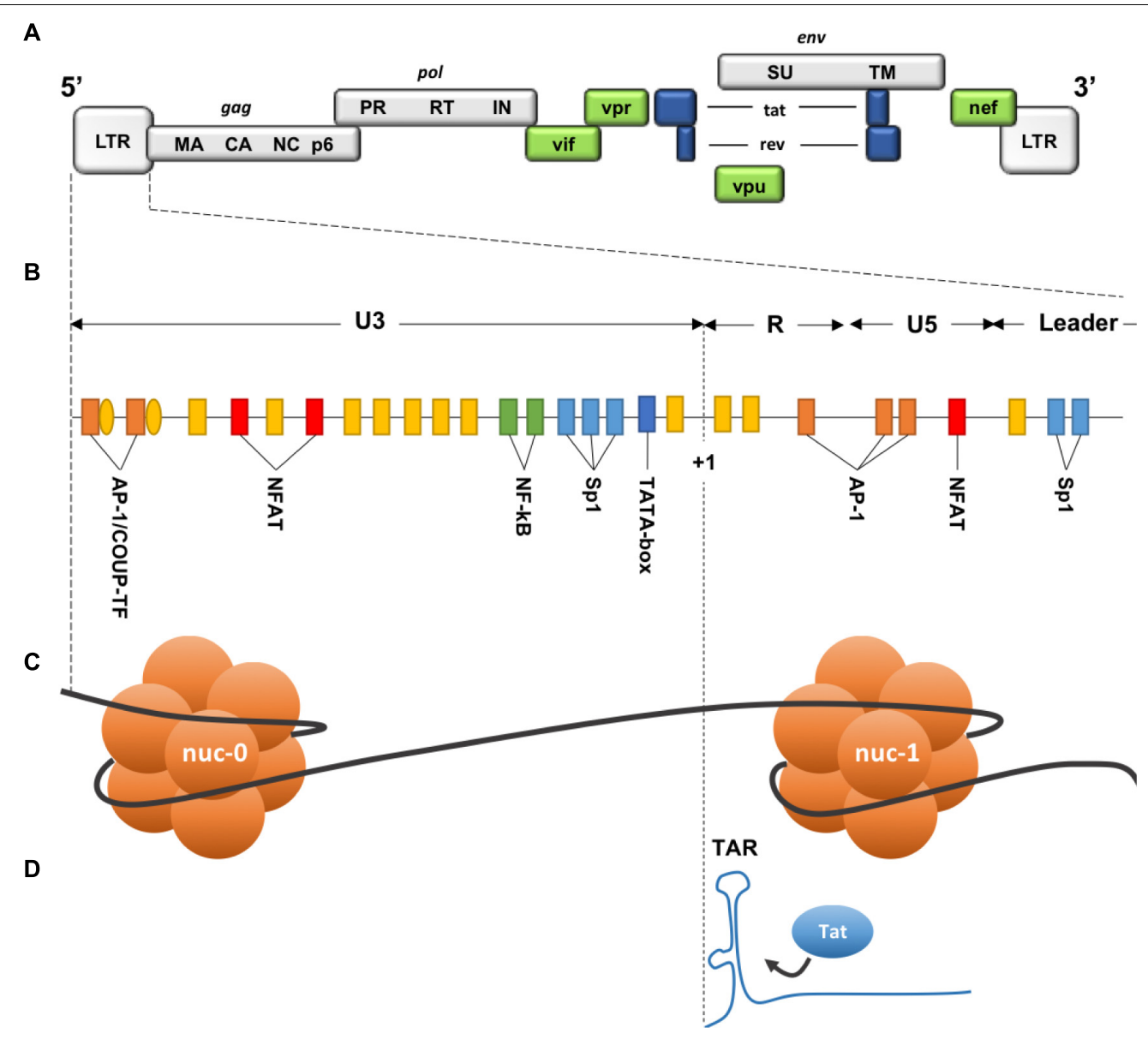

FIGURE 6 | Description of the 5' LTR of HIV-1. (A) HIV genomic structure. (B) Binding sites for cellular factors within the $5^{\prime}$ LTR. (C) Nucleosomes structure within the $5^{\prime}$ LTR. (D) Trans-activation Response element on the neosynthesized RNA. HIV genome is bordered by two identical Long Terminal Repeats (LTR $5^{\prime}$ and $\left.3^{\prime}\right)$, each composed of 3 regions: U3 (Unique $3^{\prime}$ ), R (repeated), and U5 (Unique 5'). Three functional units can be identified within U3 region: (1) a modulatory element with binding sites for transcription regulators including negative regulators. (2) an enhancer containing two binding sites for NF-kB. (3) the core promoter region comprising 3 binding sites for specificity protein 1 (Sp1) and a TATA box. R region contains the transactivation response element TAR, an RNA stem-loop at the $5^{\prime}$ end of the neo-synthesized strand, necessary for Tat-dependent transcription and elongation block alleviation, while U5 region contains additional modulatory/regulatory sites. Nucleosome nuc- 1 is located immediately after the transcription start site $(+1)$, chromatin remodeling is required to overcome this second elongation block. (Adapted from Colin and Van Lint, 2009).

Marini et al., 2015). Unexpectedly, the same integration pattern was observed in resting $\mathrm{CD} 4+\mathrm{T}$ cells from infected patients under cART (Han et al., 2004). Both defective proviruses and transcriptional interference due to close host's genes promoters were proposed to explain this non-productive state (Han et al., 2008; Lenasi et al., 2008). In addition, sensitivity to nearby heterochromatin may contribute to the silencing of HIV promoter. Indeed, in different cellular clones, heterogeneity of basal expression of HIV promoter depending on the integration site was observed (Jordan, 2001), and although less than $1 \%$ of integration events are thought to directly lead to latency (Schröder et al., 2002), integration within or near heterochromatin regions resulted in latent proviruses (Jordan et al., 2003; Sherrill-Mix et al., 2013; Battivelli et al., 2018). Moreover, following global activation signals, latently infected Jurkat clones (J-Lat) showed a variegated reactivation of HIV promoter and a different rate of de-activation once the signal was withdrawn, likely due to different integration sites (Jordan et al., 2003). Same position effect variegation following reactivation signals was linked to variable distances from enhancer sequences and repressive chromatin (Chen et al., 2016). Of note, regulatory elements can impact genes hundreds of kilobases away through various interactions allowed by the spatial genome organization (Gibcus and Dekker, 2013), distant interactions such as gene looping can also allow heterochromatin spreading and the silencing of active genes (Talbert and Henikoff, 2006). Even though the integration context alone is not sufficient to predict the fate of infection, it is an additional factor that contributes to both the establishment and reversal of latency.

Two nucleosomes (nuc-0 and nuc-1) form within the promoter region of the $5^{\prime}$ LTR of HIV provirus (Figure 6), nuc-1 is of most importance as it is located immediately downstream of the transcription start site and therefore constitute an additional elongation block (Verdin et al., 1993). To overcome this 
block and allow HIV-1 promoter activation, nuc-1 remodeling by SWItch/Sucrose Non-Fermentable (SWI/SNF) complex is required (El Kharroubi et al., 1998; Agbottah et al., 2006; Mahmoudi et al., 2006; Tréand et al., 2006; Rafati et al., 2011). Maintenance of this nuc-1 repressive structure was also associated with histone deacetylation, more precisely the recruitment by several transcription factors of the histone deacetylase 1 (HDAC1) (Van Lint et al., 1996; Sheridan et al., 1997; Coull et al., 2000; He and Margolis, 2002; Lusic et al., 2003). Other histone modifications are involved in HIV-1 LTR repression such as trimethylation of the Lysine 9 or 27 of the histone 3 (H3K9me3 and H3K27me3). Suv39H1, a histone lysine methyl transferase (HKMT), responsible for H3K9 trimethylation, is recruited on the viral promoter by the negative factor CTIP2. HP1 binds the H3K9me3 repressive marks and subsequently recruits additional Suv39H1, this selfsustaining loop is thought to allow the spread and maintenance of heterochromatin, hence reducing DNA access to transcription factors (du Chéné et al., 2007, 39; Marban et al., 2007). Additional HKMTs were shown to participate in HIV-1 LTR silencing, such as G9a/GLP (Imai et al., 2010) and very recently SETDB1 through its interaction with the HUSH complex (Tchasovnikarova et al., 2015).

Human Silencing Hub complex activity was demonstrated on different cellular and viral promoters including the Murine Leukemia Virus (MLV) and HIV-1 LTRs (Tchasovnikarova et al., 2015). In J-Lat cells, HUSH depletion resulted in promoter reactivation and GFP expression, following a loss of H3K9me3 marks on the provirus, this effect was, however, clone-dependent as some clones did not reactivate after HUSH depletion (Tchasovnikarova et al., 2015). Indeed, Tchasovnikarova et al. (2015) found the HUSH complex to localize only on a specific group of endogenous genomic loci. Here again, the integration site appears critical as it determines the silencing ability of the HUSH complex.

Whether HIV latency is a byproduct of infection, a viral strategy to persist or on the contrary a cellular defense mechanism, remains to be determined. Overall, accumulating evidence pictures HIV latency as a multifactorial phenomenon that includes several control layers, that are probably overlapping and interacting but most importantly that are dynamic and responsive to environmental changes.

\section{Strategies to Address Latency: "Speak Now or Forever Hold Your Peace"}

For more than 30 years, huge efforts have been mobilized in finding a cure for HIV, from vaccines to gene therapies, every available option has been investigated. To date, only one attempt at curing an HIV-1 positive patient revealed successful, namely the Berlin patient (Timothy Ray Brown) (Hütter et al., 2009). Diagnosed with an acute myeloid leukemia (AML), the Berlin patient received high doses of whole-body irradiation and two bone-marrow transplants from a homozygous CCR5 $\Delta 32$ donor, providing resistance against HIV-1 infection. Since then, the Berlin patient showed no detectable signs of viral rebound for more than 5 years after cART interruption (Yukl et al., 2013). Unfortunately, attempts at reproducing this "sterilizing cure" on other seropositive patients failed, as viral rebound was observed as well as cases of drug- resistance and HIV tropism shift from CCR5 to CXCR4 co-receptor (Henrich et al., 2014; Kordelas et al., 2014). Latent reservoirs are thought to play a critical role preventing viral clearance by providing a constant pool of replicative competent viruses. Hence, two diametrically opposed strategies are envisioned to deal with what might be the last hurdle to HIV cure: purging the reservoirs, as proposed by the "shock and kill" strategy or on the contrary inducing a permanent viral control in the absence of therapy, in the case of a "functional cure" strategy.

\section{Shock and Kill}

The "shock and kill" strategy was initially based on two observations: (i) the capacity of latent reservoirs to reactivate and shift from latent to productive infection, (ii) the cytopathic effect of viral replication and the killing of infected cells by the immune system (Archin et al., 2012a; Deeks, 2012). Activation of HIV expression in latent reservoirs is therefore expected to trigger elimination of HIV infected cells, while HIV dissemination would be prevented by cART. In this scope, several latency reversal agents (LRA) have been characterized including immune modulators [antibodies targeting immune checkpoints such as anti-PD-1 (Evans et al., 2018)], P-TEFb activators, protein kinase $\mathrm{C}$ (PKC) activators that induce NF- $\mathrm{KB}$ (bryostatin-1, prostatin and Ingenol) and inhibitors of chromatin-modifiers [such as HDAC inhibitors (HDACi) and histone methyltransferase inhibitors (HMTi)]. Although some of these LRAs show a slight increase of plasma RNA levels in clinical trials, none seems to effectively shrink the reservoir size (Elliott et al., 2014, 2015; Rasmussen et al., 2014; Søgaard et al., 2015; Spivak et al., 2015).

Besides the complexity of accurate quantification of the size of the reservoir, growing evidence indicate that given the heterogeneity of the latently infected cells, only a small proportion seems responsive to LRAs (Ho et al., 2013; Chen et al., 2017; Battivelli et al., 2018). Therefore, as with cART, combination of various LRAs would probably be required to achieve an effective "shock." In addition, viral-induced cytopathic effect alone was found insufficient to induce the "kill" phase which implies that beforehand immune stimulation will be needed (Shan et al., 2012; Denton et al., 2014; Deng et al., 2015). Considered strategies to optimize reservoir elimination include broadly neutralizing antibodies, therapeutic vaccines and immune modulators, reviewed in Kim et al. (2018). However, multiplying drugs raises concern over toxicity and the impact of non-specific effects on gene expression (Walker-Sperling et al., 2016; Dental et al., 2017; White et al., 2018), thus requiring an equilibrium between efficacy and safety.

\section{Functional Cure}

"HIV controllers" or "Elite controllers" are a rare population of HIV infected patients $(<1 \%)$ who are able to spontaneously maintain their plasma viremia to almost undetectable levels. The precise mechanism responsible for this viral control is unknown but seems to result from several factors, including patient genetic backgrounds (Cockerham and Hatano, 2015). Based on the characteristics of these unique populations, the "functional cure" 
strategy aims at reaching durable remission in the absence of viral eradication. In fact, long-term remission was proved possible if therapy was initiated early during primary infection. Indeed, the "Mississippi child" born from a seropositive mother and who received cART $30 \mathrm{~h}$ after birth, remained with undetectable viral loads for 2 years after treatment interruption (Persaud et al., 2013). These results were confirmed with the VISCONTI cohort in which 14 patients reached long-term remission during a median period of 7 years after treatment interruption (SáezCirión et al., 2013). Recently, a 12 years remission was also reported in a teen infected at birth and treated before 6 months of age (Frange et al., 2016).

Unfortunately, even under virological suppression, signs of ongoing viral replication were reported (Persaud et al., 2004; Fletcher et al., 2014; Lorenzo-Redondo et al., 2016). Remission can therefore last for long periods of time but eventually viral loads increase and therapeutic intervention is soon necessary. Consequently, recent strategies aim at providing more than viral control but rather a permanent inhibition of viral expression, thus preventing reservoirs' reactivation through the establishment of a "deep latency" state.

Due to its crucial role in the efficient activation of HIV transcription, the viral protein Tat is often targeted by such strategies. For instance, an analog of cortistatin A, didehydrocortistatin A (dCA), was shown to inhibit HIV Tat-dependent transcription by interacting with the TAR-binding domain of Tat, hence greatly reducing viral reactivation in response to stimulus (Mousseau et al., 2012, 2015). Another Tat inhibitor, Triptolide, is currently under clinal trial for its impact on reservoirs. Already used for rheumatoid arthritis, Triptolide was also reported to induce Tat degradation (Wan and Chen, 2014). Finally, this time through the inhibition of LEDGF/p75 interaction with the viral integrase, an interesting strategy proposes to avoid viral reactivation by directing HIV integration into transcriptionally inactive regions (Le Rouzic et al., 2013; Vranckx et al., 2016).

In any event, such therapies can only be delivered in combination with cART, at least during primary infection, and their potential in delaying or impeding viral rebound in patients remains to be demonstrated.

\section{What HUSH Antagonism by Vpx May Tell Us About HIV Cure Strategies?}

In the context of "the shock and kill" strategy, the HUSH complex is a new factor to consider. It appears interesting to address the impact of HUSH complex inactivation on latently infected cells from patients, either through Vpx delivery or drug development. More importantly, HUSH antagonism by Vpx may have even deeper implications on our understanding of latency. Indeed, inactivation of an epigenetic regulator by a viral protein, further

\section{REFERENCES}

Agbottah, E., Deng, L., Dannenberg, L. O., Pumfery, A., and Kashanchi, F. (2006). Effect of SWI/SNF chromatin remodeling complex on HIV-1 Tat activated transcription. Retrovirology 3:48. doi: 10.1186/1742-4690-3-48 strengthens the idea of latency as a cellular defense mechanism, protecting genomic and proteomic integrity.

Hepatitis $B$ virus $\mathrm{x}$ protein $(\mathrm{HBx})$ was previously found to prevent H3K9me3 deposit by SETDB1 on the covalently closed circular HBV DNA (cccDNA) (Rivière et al., 2015), but the exact mechanism remains unknown. In our study, Vpx specifically binds and induces the degradation of an epigenetic regulator, Vpx thus identifies HUSH complex and the epigenetic machinery as part of the intrinsic immunity. The "shock and kill" strategy therefore may appear in opposition to the cellular defense mechanism with considerable risks due to the possible global epigenetic modifications. For instance, LINE-1 elements are also under the control of HUSH and transposition of LINE-1 can be responsible for disease as reviewed in Hancks and Kazazian (2016). On the contrary, "the block and lock" strategy, which aims at creating a state of "deep latency," might be a better option to control viral expression, though with the drawback of a possible chronic immune activation. Finally, Axonal CharcotMarie-Tooth (CMT) disease is a neurological disorder which was recently linked to the hyperactivation of the HUSH complex (Tchasovnikarova et al., 2017). This hyperactivation was shown to result from a mutation on the ATPase domain of MORC2 (R252W) (Tchasovnikarova et al., 2017). In case MORC2 is found to participate in the repression of HIV and that the sequence specificity of HUSH complex is proved, then it would be tempting to mimic such hyper activation through drug development, in order to force permanent silencing of exogenous elements including HIV.

\section{AUTHOR CONTRIBUTIONS}

GC and FM-G wrote this review, with, at the starting point, the thesis manuscript of GC. GC made the figures.

\section{FUNDING}

Work in FM-G lab is supported by grants from the "Agence Nationale de la Recherche sur le SIDA et les hépatites virales" (ANRS), SIDACTION, "Fondation de France" and "Fondation pour la Recherche Médicale" (FRM, Grant Number DEQ20140329528 attributed to FM-G). GC received a fellowship from the French government.

\section{ACKNOWLEDGMENTS}

We would like to thank Lucie Etienne, Soundasse Munir-Matloob, Michaël Martin, and Roy Matkovic for fruitful discussions.

Ahn, J., Vu, T., Novince, Z., Guerrero-Santoro, J., Rapic-Otrin, V., and Gronenborn, A. M. (2010). HIV-1 Vpr loads uracil DNA glycosylase-2 onto DCAF1, a substrate recognition subunit of a cullin 4A-RING E3 ubiquitin ligase for proteasome-dependent degradation. J. Biol. Chem. 285, 37333-37341. doi: 10.1074/jbc.M110.133181 
Akari, H., Sakuragi, J., Takebe, Y., Tomonaga, K., Kawamura, M., Fukasawa, M., et al. (1992). Biological characterization of human immunodeficiency virus type 1 and type 2 mutants in human peripheral blood mononuclear cells. Arch. Virol. 123, 157-167. doi: 10.1007/BF01317146

Archin, N. M., Liberty, A., Kashuba, A., Choudhary, S., Kuruc, J., Crooks, A., et al. (2012a). Administration of vorinostat disrupts HIV-1 latency in patients on antiretroviral therapy. Nature 487, 482-485. doi: 10.1038/nature 11286

Archin, N. M., Vaidya, N. K., Kuruc, J. D., Liberty, A. L., Wiegand, A., Kearney, M. F., et al. (2012b). Immediate antiviral therapy appears to restrict resting $\mathrm{CD}^{+}{ }^{+}$cell HIV-1 infection without accelerating the decay of latent infection. Proc. Natl. Acad. Sci. U.S.A. 109, 9523-9528. doi: 10.1073/pnas.1120248109

Baldauf, H.-M., Pan, X., Erikson, E., Schmidt, S., Daddacha, W., Burggraf, M., et al. (2012). SAMHD1 restricts HIV-1 infection in resting CD4(+) T cells. Nat. Med. 18, 1682-1687. doi: 10.1038/nm.2964

Baldauf, H.-M., Stegmann, L., Schwarz, S.-M., Ambiel, I., Trotard, M., Martin, M., et al. (2017). Vpx overcomes a SAMHD1-independent block to HIV reverse transcription that is specific to resting CD4 T cells. Proc. Natl. Acad. Sci. U.S.A. 114, 2729-2734. doi: 10.1073/pnas.1613635114

Battivelli, E., Dahabieh, M. S., Abdel-Mohsen, M., Svensson, J. P., Tojal Da Silva, I., Cohn, L. B., et al. (2018). Distinct chromatin functional states correlate with HIV latency reactivation in infected primary $\mathrm{CD}^{+} \mathrm{T}$ cells. eLife 7:e34655. doi: $10.7554 /$ eLife. 34655

Beer, B. E., Foley, B. T., Kuiken, C. L., Tooze, Z., Goeken, R. M., Brown, C. R., et al. (2001). Characterization of novel simian immunodeficiency viruses from red-capped mangabeys from Nigeria (SIVrcmNG409 and -NG411). J. Virol. 75, 12014-12027. doi: 10.1128/JVI.75.24.12014-12027.2001

Belshan, M., Kimata, J. T., Brown, C., Cheng, X., McCulley, A., Larsen, A., et al. (2012). Vpx is critical for SIVmne infection of pigtail macaques. Retrovirology 9:32. doi: 10.1186/1742-4690-9-32

Bergamaschi, A., Ayinde, D., David, A., Le Rouzic, E., Morel, M., Collin, G., et al. (2009). The human immunodeficiency virus type $2 \mathrm{Vpx}$ protein usurps the CUL4A-DDB1 DCAF1 ubiquitin ligase to overcome a postentry block in macrophage infection. J. Virol. 83, 4854-4860. doi: 10.1128/JVI.00187-09

Bhargava, S., Cox, B., Polydorou, C., Gresakova, V., Korinek, V., Strnad, H., et al. (2017). The epigenetic modifier Fam208a is required to maintain epiblast cell fitness. Sci. Rep. 7:9322. doi: 10.1038/s41598-017-09490-w

Binka, M., Ooms, M., Steward, M., and Simon, V. (2012). The activity spectrum of Vif from multiple HIV-1 subtypes against APOBEC3G, APOBEC3F, and APOBEC3H. J. Virol. 86, 49-59. doi: 10.1128/JVI.06082-11

Black, L. R., and Aiken, C. (2010). TRIM5 $\alpha$ disrupts the structure of assembled HIV-1 capsid complexes in vitro. J. Virol. 84, 6564-6569. doi: 10.1128/JVI. 00210-10

Brown, P. O., Bowerman, B., Varmus, H. E., and Bishop, J. M. (1987). Correct integration of retroviral DNA in vitro. Cell 49, 347-356. doi: 10.1016/00928674(87)90287-X

Budhiraja, S., Famiglietti, M., Bosque, A., Planelles, V., and Rice, A. P. (2013). Cyclin T1 and CDK9 T-loop phosphorylation are downregulated during establishment of HIV-1 latency in primary resting memory $\mathrm{CD}^{+} \mathrm{T}$ cells. J. Virol. 87, 1211-1220. doi: 10.1128/JVI.02413-12

Buzon, M. J., Martin-Gayo, E., Pereyra, F., Ouyang, Z., Sun, H., Li, J. Z., et al. (2014). Long-term antiretroviral treatment initiated at primary HIV-1 infection affects the size, composition, and decay kinetics of the reservoir of HIV-1-infected CD4 T cells. J. Virol. 88, 10056-10065. doi: 10.1128/JVI.01046-14

Carthagena, L., Parise, M. C., Ringeard, M., Chelbi-Alix, M. K., Hazan, U., and Nisole, S. (2008). Implication of TRIM alpha and TRIMCyp in interferoninduced anti-retroviral restriction activities. Retrovirology 5:59. doi: 10.1186/ 1742-4690-5-59

Chang, Y., Horton, J. R., Bedford, M. T., Zhang, X., and Cheng, X. (2011). Structural insights for MPP8 chromodomain interaction with histone $\mathrm{H} 3$ lysine 9: potential effect of phosphorylation on methyl-lysine binding. J. Mol. Biol. 408, 807-814. doi: 10.1016/j.jmb.2011.03.018

Chen, H.-C., Martinez, J. P., Zorita, E., Meyerhans, A., and Filion, G. J. (2016). Position effects influence HIV latency reversal. Nat. Struct. Mol. Biol. 24, 47-54. doi: $10.1038 / \mathrm{nsmb} .3328$

Chen, H.-C., Martinez, J. P., Zorita, E., Meyerhans, A., and Filion, G. J. (2017). Position effects influence HIV latency reversal. Nat. Struct. Mol. Biol. 24, 47-54. doi: $10.1038 / \mathrm{nsmb} .3328$
Chomont, N., El-Far, M., Ancuta, P., Trautmann, L., Procopio, F. A., YassineDiab, B., et al. (2009). HIV reservoir size and persistence are driven by $\mathrm{T}$ cell survival and homeostatic proliferation. Nat. Med. 15, 893-900. doi: 10.1038/ nm.1972

Chougui, G., Munir-Matloob, S., Matkovic, R., Martin, M. M., Morel, M., Lahouassa, H., et al. (2018). HIV-2/SIV viral protein X counteracts HUSH repressor complex. Nat. Microbiol. 3, 891-897. doi: 10.1038/s41564-018-0179-6

Chun, T. W., Carruth, L., Finzi, D., Shen, X., DiGiuseppe, J. A., Taylor, H., et al. (1997). Quantification of latent tissue reservoirs and total body viral load in HIV-1 infection. Nature 387, 183-188. doi: 10.1038/387183a0

Chun, T.-W., Engel, D., Berrey, M. M., Shea, T., Corey, L., and Fauci, A. S. (1998). Early establishment of a pool of latently infected, resting CD $4^{+} \mathrm{T}$ cells during primary HIV-1 infection. Proc. Natl. Acad. Sci. U.S.A. 95, 8869-8873. doi: $10.1073 /$ pnas.95.15.8869

Cockerham, L. R., and Hatano, H. (2015). Elite control of HIV: is this the right model for a functional cure? Trends Microbiol. 23, 71-75. doi: 10.1016/j.tim. 2014.11.003

Cohen, E. A., Dehni, G., Sodroski, J. G., and Haseltine, W. A. (1990a). Human immunodeficiency virus vpr product is a virion-associated regulatory protein. J. Virol. 64, 3097-3099.

Cohen, E. A., Terwilliger, E. F., Jalinoos, Y., Proulx, J., Sodroski, J. G., and Haseltine, W. A. (1990b). Identification of HIV-1 vpr product and function. J. Acquir. Immune Defic. Syndr. 3, 11-18.

Colin, L., and Van Lint, C. (2009). Molecular control of HIV-1 postintegration latency: implications for the development of new therapeutic strategies. Retrovirology 6:111. doi: 10.1186/1742-4690-6-111

Compton, A. A., Bruel, T., Porrot, F., Mallet, A., Sachse, M., Euvrard, M., et al. (2014). IFITM proteins incorporated into HIV-1 virions impair viral fusion and spread. Cell Host Microbe 16, 736-747. doi: 10.1016/j.chom.2014.11.001

Conticello, S. G., Harris, R. S., and Neuberger, M. S. (2003). The Vif protein of HIV triggers degradation of the human antiretroviral DNA deaminase APOBEC3G. Curr. Biol. 13, 2009-2013. doi: 10.1016/j.cub.2003.10.034

Coull, J. J., Romerio, F., Sun, J. M., Volker, J. L., Galvin, K. M., Davie, J. R., et al. (2000). The human factors YY1 and LSF repress the human immunodeficiency virus type 1 long terminal repeat via recruitment of histone deacetylase 1 . J. Virol. 74, 6790-6799. doi: 10.1128/JVI.74.15.6790-6799.2000

Deeks, S. G. (2012). HIV: shock and kill. Nature 487, 439-440. doi: 10.1038/487439a Deng, K., Pertea, M., Rongvaux, A., Wang, L., Durand, C. M., Ghiaur, G., et al. (2015). Broad CTL response is required to clear latent HIV-1 due to dominance of escape mutations. Nature 517, 381-385. doi: 10.1038/nature14053

Dental, C., Proust, A., Ouellet, M., Barat, C., and Tremblay, M. J. (2017). HIV-1 latency-reversing agents prostratin and bryostatin-1 induce blood-brain barrier disruption/inflammation and modulate leukocyte adhesion/transmigration. J. Immunol. 198, 1229-1241. doi: 10.4049/jimmunol.1600742

Denton, P. W., Long, J. M., Wietgrefe, S. W., Sykes, C., Spagnuolo, R. A., Snyder, O. D., et al. (2014). Targeted cytotoxic therapy kills persisting HIV infected cells during ART. PLoS Pathog. 10:e1003872. doi: 10.1371/journal.ppat. 1003872

Descours, B., Cribier, A., Chable-Bessia, C., Ayinde, D., Rice, G., Crow, Y., et al. (2012). SAMHD1 restricts HIV-1 reverse transcription in quiescent $\mathrm{CD}^{+}$ T-cells. Retrovirology 9:87. doi: 10.1186/1742-4690-9-87

Douse, C. H., Bloor, S., Liu, Y., Shamin, M., Tchasovnikarova, I. A., Timms, R. T., et al. (2018). Neuropathic MORC2 mutations perturb GHKL ATPase dimerization dynamics and epigenetic silencing by multiple structural mechanisms. Nat. Commun. 9:651. doi: 10.1038/s41467-01803045-x

Doyle, T., Goujon, C., and Malim, M. H. (2015). HIV-1 and interferons: who's interfering with whom? Nat. Rev. Microbiol. 13, 403-413. doi: 10.1038/ nrmicro3449

du Chéné, I., Basyuk, E., Lin, Y.-L., Triboulet, R., Knezevich, A., Chable-Bessia, C., et al. (2007). Suv39H1 and HP1gamma are responsible for chromatin-mediated HIV-1 transcriptional silencing and post-integration latency. EMBO J. 26, 424-435. doi: 10.1038/sj.emboj.7601517

Duggal, N. K., and Emerman, M. (2012). Evolutionary conflicts between viruses and restriction factors shape immunity. Nat. Rev. Immunol. 12, 687-695. doi: $10.1038 /$ nri3295

Eberl, H. C., Spruijt, C. G., Kelstrup, C. D., Vermeulen, M., and Mann, M. (2013). A map of general and specialized chromatin readers in mouse tissues generated 
by label-free interaction proteomics. Mol. Cell 49, 368-378. doi: 10.1016/j. molcel.2012.10.026

El Kharroubi, A., Piras, G., Zensen, R., and Martin, M. A. (1998). Transcriptional activation of the integrated chromatin-associated human immunodeficiency virus type 1 promoter. Mol. Cell. Biol. 18, 2535-2544. doi: 10.1128/MCB.18.5. 2535

Elgin, S. C. R., and Reuter, G. (2013). Position-effect variegation, heterochromatin formation, and gene silencing in Drosophila. Cold Spring Harb. Perspect. Biol. 5:a017780. doi: 10.1101/cshperspect.a017780

Elliott, J. H., McMahon, J. H., Chang, C. C., Lee, S. A., Hartogensis, W., Bumpus, N., et al. (2015). Short-term administration of disulfiram for reversal of latent HIV infection: a phase 2 dose-escalation study. Lancet HIV 2, e520-e529. doi: 10.1016/S2352-3018(15)00226-X

Elliott, J. H., Wightman, F., Solomon, A., Ghneim, K., Ahlers, J., Cameron, M. J., et al. (2014). Activation of HIV transcription with short-course vorinostat in HIV-infected patients on suppressive antiretroviral therapy. PLoS Pathog. 10:e1004473. doi: 10.1371/journal.ppat.1004473

Evans, V. A., Van Der Sluis, R. M., Solomon, A., Dantanarayana, A., McNeil, C., Garsia, R., et al. (2018). PD-1 contributes to the establishment and maintenance of HIV-1 latency. AIDS 32, 1491-1497. doi: 10.1097/QAD.0000000000001849

Finzi, D., Blankson, J., Siliciano, J. D., Margolick, J. B., Chadwick, K., Pierson, T., et al. (1999). Latent infection of $\mathrm{CD} 4^{+} \mathrm{T}$ cells provides a mechanism for lifelong persistence of HIV-1, even in patients on effective combination therapy. Nat. Med. 5, 512-517. doi: 10.1038/8394

Fletcher, C. V., Staskus, K., Wietgrefe, S. W., Rothenberger, M., Reilly, C., Chipman, J. G., et al. (2014). Persistent HIV-1 replication is associated with lower antiretroviral drug concentrations in lymphatic tissues. Proc. Natl. Acad. Sci. U.S.A. 111, 2307-2312. doi: 10.1073/pnas.1318249111

Frange, P., Faye, A., Avettand-Fenoël, V., Bellaton, E., Descamps, D., Angin, M., et al. (2016). HIV-1 virological remission lasting more than 12 years after interruption of early antiretroviral therapy in a perinatally infected teenager enrolled in the French ANRS EPF-CO10 paediatric cohort: a case report. Lancet HIV 3, e49-e54. doi: 10.1016/S2352-3018(15)00232-5

Fujita, M., Otsuka, M., Miyoshi, M., Khamsri, B., Nomaguchi, M., and Adachi, A. (2008a). Vpx is critical for reverse transcription of the human immunodeficiency virus type 2 genome in macrophages. J. Virol. 82, 7752-7756. doi: 10.1128/JVI.01003-07

Fujita, M., Otsuka, M., Nomaguchi, M., and Adachi, A. (2008b). Functional region mapping of HIV-2 Vpx protein. Microbes Infect. 10, 1387-1392. doi: 10.1016/j. micinf.2008.08.005

Gao, D., Wu, J., Wu, Y.-T., Du, F., Aroh, C., Yan, N., et al. (2013). Cyclic GMP-AMP synthase is an innate immune sensor of HIV and other retroviruses. Science 341, 903-906. doi: 10.1126/science.1240933

Gibbs, J. S., Lackner, A. A., Lang, S. M., Simon, M. A., Sehgal, P. K., Daniel, M. D., et al. (1995). Progression to AIDS in the absence of a gene for vpr or vpx. J. Virol. 69, 2378-2383.

Gibbs, J. S., Regier, D. A., and Desrosiers, R. C. (1994). Construction and in vitro properties of SIVmac mutants with deletions in "nonessential" genes. AIDS Res. Hum. Retroviruses 10, 607-616. doi: 10.1089/aid.1994.10.607

Gibcus, J. H., and Dekker, J. (2013). The hierarchy of the 3D genome. Mol. Cell 49, 773-782. doi: 10.1016/j.molcel.2013.02.011

Global HIV and AIDS Statistics (2018). Fact Sheet. Available at: http://www.unaids. org/en/resources/fact-sheet [accessed December 29, 2018].

Goldstone, D. C., Ennis-Adeniran, V., Hedden, J. J., Groom, H. C. T., Rice, G. I., Christodoulou, E., et al. (2011). HIV-1 restriction factor SAMHD1 is a deoxynucleoside triphosphate triphosphohydrolase. Nature 480, 379-382. doi: $10.1038 /$ nature 10623

Goujon, C., Jarrosson-Wuillème, L., Bernaud, J., Rigal, D., Darlix, J.-L., and Cimarelli, A. (2006). With a little help from a friend: increasing HIV transduction of monocyte-derived dendritic cells with virion-like particles of SIVMAC. Gene Ther. 13, 991-994. doi: 10.1038/sj.gt.3302753

Goujon, C., Moncorgé, O., Bauby, H., Doyle, T., Ward, C. C., Schaller, T., et al. (2013). Human MX2 is an interferon-induced post-entry inhibitor of HIV-1 infection. Nature 502, 559-562. doi: 10.1038/nature12542

Goujon, C., Rivière, L., Jarrosson-Wuilleme, L., Bernaud, J., Rigal, D., Darlix, J.L., et al. (2007). SIVSM/HIV-2 Vpx proteins promote retroviral escape from a proteasome-dependent restriction pathway present in human dendritic cells. Retrovirology 4:2. doi: 10.1186/1742-4690-4-2
Gupta, R. K., Hué, S., Schaller, T., Verschoor, E., Pillay, D., and Towers, G. J. (2009). Mutation of a single residue renders human tetherin resistant to HIV-1 Vpu-mediated depletion. PLoS Pathog. 5:e1000443. doi: 10.1371/journal.ppat. 1000443

Guyader, M., Emerman, M., Montagnier, L., and Peden, K. (1989). VPX mutants of HIV-2 are infectious in established cell lines but display a severe defect in peripheral blood lymphocytes. EMBO J. 8, 1169-1175. doi: 10.1002/j.14602075.1989.tb03488.x

Han, Y., Lassen, K., Monie, D., Sedaghat, A. R., Shimoji, S., Liu, X., et al. (2004). Resting $\mathrm{CD}^{+} \mathrm{T}$ cells from human immunodeficiency virus type 1 (HIV-1)infected individuals carry integrated HIV-1 genomes within actively transcribed host genes. J. Virol. 78, 6122-6133. doi: 10.1128/JVI.78.12.6122-6133.2004

Han, Y., Lin, Y. B., An, W., Xu, J., Yang, H.-C., O'Connell, K., et al. (2008). Orientation-dependent regulation of integrated HIV-1 expression by host gene transcriptional readthrough. Cell Host Microbe 4, 134-146. doi: 10.1016/j.chom. 2008.06.008

Hancks, D. C., and Kazazian, H. H. (2016). Roles for retrotransposon insertions in human disease. Mob. DNA 7:9. doi: 10.1186/s13100-016-0065-9

Harris, R. S., Bishop, K. N., Sheehy, A. M., Craig, H. M., Petersen-Mahrt, S. K., Watt, I. N., et al. (2003). DNA deamination mediates innate immunity to retroviral infection. Cell 113, 803-809. doi: 10.1016/S0092-8674(03)00423-9

Harris, R. S., Hultquist, J. F., and Evans, D. T. (2012). The restriction factors of human immunodeficiency virus. J. Biol. Chem. 287, 40875-40883. doi: 10.1074/ jbc.R112.416925

Harten, S. K., Bruxner, T. J., Bharti, V., Blewitt, M., Nguyen, T.-M.-T., Whitelaw, E., et al. (2014). The first mouse mutants of D14Abble (Fam208a) show that it is critical for early development. Mamm. Genome 25, 293-303. doi: 10.1007/ s00335-014-9516-0

Hatziioannou, T., Princiotta, M., Piatak, M., Yuan, F., Zhang, F., Lifson, J. D., et al. (2006). Generation of simian-tropic HIV-1 by restriction factor evasion. Science 314:95. doi: 10.1126/science.1130994

He, G., and Margolis, D. M. (2002). Counterregulation of chromatin deacetylation and histone deacetylase occupancy at the integrated promoter of human immunodeficiency virus type 1 (HIV-1) by the HIV-1 repressor YY 1 and HIV-1 activator Tat. Mol. Cell. Biol. 22, 2965-2973. doi: 10.1128/MCB.22.9.2965-2973. 2002

He, J., Choe, S., Walker, R., Di Marzio, P., Morgan, D. O., and Landau, N. R. (1995). Human immunodeficiency virus type 1 viral protein $\mathrm{R}(\mathrm{Vpr})$ arrests cells in the G2 phase of the cell cycle by inhibiting p34cdc2 activity. J. Virol. 69, 6705-6711.

Henderson, L. E., Sowder, R. C., Copeland, T. D., Benveniste, R. E., and Oroszlan, S. (1988). Isolation and characterization of a novel protein (X-ORF product) from SIV and HIV-2. Science 241, 199-201. doi: 10.1126/science.3388031

Henrich, T. J., Hanhauser, E., Marty, F. M., Sirignano, M. N., Keating, S., Lee, T.H., et al. (2014). Antiretroviral-free HIV-1 remission and viral rebound after allogeneic stem cell transplantation: report of 2 cases. Ann. Intern. Med. 161, 319-327. doi: 10.7326/M14-1027

Hirsch, V. M., Sharkey, M. E., Brown, C. R., Brichacek, B., Goldstein, S., Wakefield, J., et al. (1998). Vpx is required for dissemination and pathogenesis of SIV(SM) PBj: evidence of macrophage-dependent viral amplification. Nat. Med. 4, 1401-1408. doi: 10.1038/3992

Ho, Y.-C., Shan, L., Hosmane, N. N., Wang, J., Laskey, S. B., Rosenbloom, D. I. S., et al. (2013). Replication-competent non-induced proviruses in the latent reservoir increase barrier to HIV-1 cure. Cell 155, 540-551. doi: 10.1016/ j.cell.2013.09.020

Hrecka, K., Hao, C., Gierszewska, M., Swanson, S. K., Kesik-Brodacka, M., Srivastava, S., et al. (2011). Vpx relieves inhibition of HIV-1 infection of macrophages mediated by the SAMHD1 protein. Nature 474, 658-661. doi: 10.1038 /nature10195

Hu, J., Switzer, W. M., Foley, B. T., Robertson, D. L., Goeken, R. M., Korber, B. T., et al. (2003). Characterization and comparison of recombinant simian immunodeficiency virus from drill (Mandrillus leucophaeus) and mandrill (Mandrillus sphinx) isolates. J. Virol. 77, 4867-4880. doi: 10.1128/JVI.77.8.48674880.2003

Hu, W., Vander Heyden, N., and Ratner, L. (1989). Analysis of the function of viral protein X (VPX) of HIV-2. Virology 173, 624-630. doi: 10.1016/0042-6822(89) 90574-6

Huh, J.-W., Kim, T.-H., Yi, J.-M., Park, E.-S., Kim, W.-Y., Sin, H.-S., et al. (2006). Molecular evolution of the periphilin gene in relation to human endogenous 
retrovirus m element. J. Mol. Evol. 62, 730-737. doi: 10.1007/s00239-0050109-0

Hütter, G., Nowak, D., Mossner, M., Ganepola, S., Müssig, A., Allers, K., et al. (2009). Long-term control of HIV by CCR5 Delta32/Delta32 stemcell transplantation. N. Engl. J. Med. 360, 692-698. doi: 10.1056/NEJMoa08 02905

Imai, K., Togami, H., and Okamoto, T. (2010). Involvement of Histone H3 Lysine 9 (H3K9) methyltransferase G9a in the maintenance of hiv-1 latency and its reactivation by BIX01294. J. Biol. Chem. 285, 16538-16545. doi: 10.1074/jbc. M110.103531

Jakobsen, M. R., Bak, R. O., Andersen, A., Berg, R. K., Jensen, S. B., Tengchuan, J., et al. (2013). IFI16 senses DNA forms of the lentiviral replication cycle and controls HIV-1 replication. Proc. Natl. Acad. Sci. U.S.A. 110, E4571-E4580. doi: $10.1073 /$ pnas. 1311669110

Jordan, A. (2001). The site of HIV-1 integration in the human genome determines basal transcriptional activity and response to Tat transactivation. EMBO J. 20, 1726-1738. doi: 10.1093/emboj/20.7.1726

Jordan, A., Bisgrove, D., and Verdin, E. (2003). HIV reproducibly establishes a latent infection after acute infection of T cells in vitro. EMBO J. 22, 1868-1877. doi: $10.1093 /$ emboj/cdg188

Jowett, J. B., Planelles, V., Poon, B., Shah, N. P., Chen, M. L., and Chen, I. S. (1995). The human immunodeficiency virus type 1 vpr gene arrests infected T cells in the $\mathrm{G}^{+}{ }^{+} \mathrm{M}$ phase of the cell cycle. J. Virol. 69, 6304-6313.

Kamada, K., Igarashi, T., Martin, M. A., Khamsri, B., Hatcho, K., Yamashita, T., et al. (2006). Generation of HIV-1 derivatives that productively infect macaque monkey lymphoid cells. Proc. Natl. Acad. Sci. U.S.A. 103, 16959-16964. doi: 10.1073/pnas.0608289103

Kane, M., Yadav, S. S., Bitzegeio, J., Kutluay, S. B., Zang, T., Wilson, S. J., et al. (2013). Mx2 is an interferon induced inhibitor of HIV-1 infection. Nature 502, 563-566. doi: 10.1038/nature12653

Kao, S. Y., Calman, A. F., Luciw, P. A., and Peterlin, B. M. (1987). Anti-termination of transcription within the long terminal repeat of HIV-1 by tat gene product. Nature 330, 489-493. doi: 10.1038/330489a0

Kappes, J. C., Conway, J. A., Lee, S. W., Shaw, G. M., and Hahn, B. H. (1991). Human immunodeficiency virus type 2 vpx protein augments viral infectivity. Virology 184, 197-209. doi: 10.1016/0042-6822(91)90836-Z

Kappes, J. C., Morrow, C. D., Lee, S. W., Jameson, B. A., Kent, S. B., Hood, L. E., et al. (1988). Identification of a novel retroviral gene unique to human immunodeficiency virus type 2 and simian immunodeficiency virus SIVMAC. J. Virol. 62, 3501-3505.

Kawamura, M., Sakai, H., and Adachi, A. (1994). Human immunodeficiency virus $\mathrm{Vpx}$ is required for the early phase of replication in peripheral blood mononuclear cells. Microbiol. Immunol. 38, 871-878. doi: 10.1111/j.1348-0421. 1994.tb02140.x

Kim, Y., Anderson, J. L., and Lewin, S. R. (2018). Getting the "Kill" into "Shock and Kill": strategies to eliminate latent HIV. Cell Host Microbe 23, 14-26. doi: 10.1016/j.chom.2017.12.004

Kokura, K., Sun, L., Bedford, M. T., and Fang, J. (2010). Methyl-H3K9-binding protein MPP8 mediates E-cadherin gene silencing and promotes tumour cell motility and invasion. EMBO J. 29, 3673-3687. doi: 10.1038/emboj. 2010.239

Kordelas, L., Verheyen, J., Beelen, D. W., Horn, P. A., Heinold, A., Kaiser, R., et al. (2014). Shift of HIV tropism in stem-cell transplantation with CCR5 Delta32 mutation. N. Engl. J. Med. 371, 880-882. doi: 10.1056/NEJMc1405805

Lafuse, W. P., Brown, D., Castle, L., and Zwilling, B. S. (1995). Cloning and characterization of a novel cDNA that is IFN-gamma-induced in mouse peritoneal macrophages and encodes a putative GTP-binding protein. J. Leukoc. Biol. 57, 477-483. doi: 10.1002/jlb.57.3.477

Laguette, N., Rahm, N., Sobhian, B., Chable-Bessia, C., Münch, J., Snoeck, J., et al. (2012). Evolutionary and functional analyses of the interaction between the myeloid restriction factor SAMHD1 and the lentiviral Vpx protein. Cell Host Microbe 11, 205-217. doi: 10.1016/j.chom.2012.01.007

Laguette, N., Sobhian, B., Casartelli, N., Ringeard, M., Chable-Bessia, C., Ségéral, E., et al. (2011). SAMHD1 is the dendritic- and myeloid-cell-specific HIV-1 restriction factor counteracted by Vpx. Nature 474, 654-657. doi: 10.1038/ nature 10117

Lahaye, X., Satoh, T., Gentili, M., Cerboni, S., Conrad, C., Hurbain, I., et al. (2013). The capsids of HIV-1 and HIV-2 determine immune detection of the viral
cDNA by the innate sensor cGAS in dendritic cells. Immunity 39, 1132-1142. doi: 10.1016/j.immuni.2013.11.002

Lahouassa, H., Daddacha, W., Hofmann, H., Ayinde, D., Logue, E. C., Dragin, L., et al. (2012). SAMHD1 restricts the replication of human immunodeficiency virus type 1 by depleting the intracellular pool of deoxynucleoside triphosphates. Nat. Immunol. 13, 223-228. doi: 10.1038/ni. 2236

Le Rouzic, E., Belaïdouni, N., Estrabaud, E., Morel, M., Rain, J.-C., Transy, C., et al. (2007). HIV1 Vpr arrests the cell cycle by recruiting DCAF1/VprBP, a receptor of the Cul4-DDB1 ubiquitin ligase. Cell Cycle 6, 182-188. doi: 10.4161/cc.6.2. 3732

Le Rouzic, E., Bonnard, D., Chasset, S., Bruneau, J.-M., Chevreuil, F., Le Strat, F., et al. (2013). Dual inhibition of HIV-1 replication by integrase-LEDGF allosteric inhibitors is predominant at the post-integration stage. Retrovirology 10:144. doi: 10.1186/1742-4690-10-144

Lenasi, T., Contreras, X., and Peterlin, B. M. (2008). Transcriptional interference antagonizes proviral gene expression to promote HIV latency. Cell Host Microbe 4, 123-133. doi: 10.1016/j.chom.2008.05.016

Li, D.-Q., Nair, S. S., Ohshiro, K., Kumar, A., Nair, V. S., Pakala, S. B., et al. (2012). MORC2 signaling facilitates phosphorylation-dependent, ATPasecoupled chromatin remodeling during the DNA damage response. Cell Rep. 2 , 1657-1669. doi: 10.1016/j.celrep.2012.11.018

Li, J., Li, Z., Ruan, J., Xu, C., Tong, Y., Pan, P. W., et al. (2011). Structural basis for specific binding of human MPP8 chromodomain to histone $\mathrm{H} 3$ methylated at lysine 9. PLoS One 6:e25104. doi: 10.1371/journal.pone.0025104

Li, N., Zhang, W., and Cao, X. (2000). Identification of human homologue of mouse IFN-gamma induced protein from human dendritic cells. Immunol. Lett. 74, 221-224. doi: 10.1016/S0165-2478(00)00276-5

Lilly, F. (1970). Fv-2: identification and location of a second gene governing the spleen focus response to Friend leukemia virus in mice. J. Natl. Cancer Inst. 45, $163-169$.

Lim, E. S., Fregoso, O. I., McCoy, C. O., Matsen, F. A., Malik, H. S., and Emerman, M. (2012). The ability of primate lentiviruses to degrade the monocyte restriction factor SAMHD1 preceded the birth of the viral accessory protein Vpx. Cell Host Microbe 11, 194-204. doi: 10.1016/j.chom.2012.01.004

Lim, E. S., Malik, H. S., and Emerman, M. (2010). Ancient adaptive evolution of tetherin shaped the functions of $\mathrm{Vpu}$ and Nef in human immunodeficiency virus and primate lentiviruses. J. Virol. 84, 7124-7134. doi: 10.1128/JVI.0046810

Liu, B., Sarkis, P. T. N., Luo, K., Yu, Y., and Yu, X.-F. (2005). Regulation of Apobec3F and human immunodeficiency virus type 1 Vif by Vif-Cul5-ElonB/C E3 ubiquitin ligase. J. Virol. 79, 9579-9587. doi: 10.1128/JVI.79.15.9579-9587. 2005

Liu, L., Oliveira, N. M., Cheney, K. M., Pade, C., Dreja, H., Bergin, A.-M. H., et al. (2011). A whole genome screen for HIV restriction factors. Retrovirology 8:94. doi: 10.1186/1742-4690-8-94

Liu, Z., Pan, Q., Ding, S., Qian, J., Xu, F., Zhou, J., et al. (2013). The interferoninducible MxB protein inhibits HIV-1 infection. Cell Host Microbe 14, 398-410. doi: 10.1016/j.chom.2013.08.015

Llano, M., Saenz, D. T., Meehan, A., Wongthida, P., Peretz, M., Walker, W. H., et al. (2006). An essential role for LEDGF/p75 in HIV integration. Science 314, 461-464. doi: 10.1126/science.1132319

Lorenzo-Redondo, R., Fryer, H. R., Bedford, T., Kim, E.-Y., Archer, J., Pond, S. L. K., et al. (2016). Persistent HIV-1 replication maintains the tissue reservoir during therapy. Nature 530, 51-56. doi: 10.1038/nature16933

Lu, J., Pan, Q., Rong, L., He, W., Liu, S.-L., and Liang, C. (2011). The IFITM proteins inhibit HIV-1 infection. J. Virol. 85, 2126-2137. doi: 10.1128/JVI. 01531- 10

Lupo, A., Cesaro, E., Montano, G., Zurlo, D., Izzo, P., and Costanzo, P. (2013). KRAB-zinc finger proteins: a repressor family displaying multiple biological functions. Curr. Genomics 14, 268-278. doi: 10.2174/13892029113149990002

Lusic, M., Marcello, A., Cereseto, A., and Giacca, M. (2003). Regulation of HIV1 gene expression by histone acetylation and factor recruitment at the LTR promoter. EMBO J. 22, 6550-6561. doi: 10.1093/emboj/cdg631

Mahmoudi, T., Parra, M., Vries, R. G. J., Kauder, S. E., Verrijzer, C. P., Ott, M., et al. (2006). The SWI/SNF chromatin-remodeling complex is a cofactor for Tat transactivation of the HIV promoter. J. Biol. Chem. 281, 19960-19968. doi: 10.1074/jbc.M603336200 
Malim, M. H., and Bieniasz, P. D. (2012). HIV restriction factors and mechanisms of evasion. Cold Spring Harb. Perspect. Med. 2:a006940. doi: 10.1101/ cshperspect.a006940

Mangeat, B., Turelli, P., Caron, G., Friedli, M., Perrin, L., and Trono, D. (2003). Broad antiretroviral defence by human APOBEC3G through lethal editing of nascent reverse transcripts. Nature 424, 99-103. doi: 10.1038/nature 01709

Marban, C., Suzanne, S., Dequiedt, F., de Walque, S., Redel, L., Van Lint, C., et al. (2007). Recruitment of chromatin-modifying enzymes by CTIP2 promotes HIV-1 transcriptional silencing. EMBO J. 26, 412-423. doi: 10.1038/sj.emboj. 7601516

Margolis, D. M. (2010). Mechanisms of HIV latency: an emerging picture of complexity. Curr. HIV AIDS Rep. 7, 37-43. doi: 10.1007/s11904-0090033-9

Mariani, R., Chen, D., Schröfelbauer, B., Navarro, F., König, R., Bollman, B., et al. (2003). Species-specific exclusion of APOBEC3G from HIV1 virions by Vif. Cell 114, 21-31. doi: 10.1016/S0092-8674(03) 00515-4

Marini, B., Kertesz-Farkas, A., Ali, H., Lucic, B., Lisek, K., Manganaro, L., et al. (2015). Nuclear architecture dictates HIV-1 integration site selection. Nature 521, 227-231. doi: 10.1038/nature14226

Martinez-Steele, E., Awasana, A. A., Corrah, T., Sabally, S., van der Sande, M., Jaye, A., et al. (2007). Is HIV-2- induced AIDS different from HIV-1-associated AIDS? Data from a West African clinic. AIDS 21, 317-324. doi: 10.1097/QAD. 0b013e328011d7ab

Matheson, N. J., Sumner, J., Wals, K., Rapiteanu, R., Weekes, M. P., Vigan, R., et al. (2015). Cell surface proteomic map of HIV infection reveals antagonism of amino acid metabolism by Vpu and Nef. Cell Host Microbe 18, 409-423. doi: 10.1016/j.chom.2015.09.003

McNatt, M. W., Zang, T., Hatziioannou, T., Bartlett, M., Fofana, I. B., Johnson, W. E., et al. (2009). Species-specific activity of HIV-1 Vpu and positive selection of tetherin transmembrane domain variants. PLoS Pathog. 5:e1000300. doi: 10.1371/journal.ppat. 1000300

Mehle, A., Strack, B., Ancuta, P., Zhang, C., McPike, M., and Gabuzda, D. (2004). Vif overcomes the innate antiviral activity of APOBEC3G by promoting its degradation in the ubiquitin-proteasome pathway. J. Biol. Chem. 279, 77927798. doi: 10.1074/jbc.M313093200

Morellet, N., Bouaziz, S., Petitjean, P., and Roques, B. P. (2003). NMR structure of the HIV-1 regulatory protein VPR. J. Mol. Biol. 327, 215-227. doi: 10.1016/ S0022-2836(03)00060-3

Mousseau, G., Clementz, M. A., Bakeman, W. N., Nagarsheth, N., Cameron, M., Shi, J., et al. (2012). An analog of the natural steroidal alkaloid cortistatin a potently suppresses tat-dependent HIV transcription. Cell Host Microbe 12, 97-108. doi: 10.1016/j.chom.2012.05.016

Mousseau, G., Kessing, C. F., Fromentin, R., Trautmann, L., Chomont, N., and Valente, S. T. (2015). The tat inhibitor didehydro-cortistatin A prevents HIV-1 reactivation from latency. $m$ Bio 6:e00465. doi: 10.1128/mBio.00465-15

Murrell, B., Vollbrecht, T., Guatelli, J., and Wertheim, J. O. (2016). The evolutionary histories of antiretroviral proteins SERINC3 and SERINC5 do not support an evolutionary arms race in primates. J. Virol. 90, 8085-8089. doi: 10.1128/JVI.00972-16

Neil, S. J. D., Zang, T., and Bieniasz, P. D. (2008). Tetherin inhibits retrovirus release and is antagonized by HIV-1 Vpu. Nature 451, 425-430. doi: 10.1038/ nature 06553

Nyamweya, S., Hegedus, A., Jaye, A., Rowland-Jones, S., Flanagan, K. L., and Macallan, D. C. (2013). Comparing HIV-1 and HIV-2 infection: lessons for viral immunopathogenesis. Rev. Med. Virol. 23, 221-240. doi: 10.1002/rmv.1739

Park, I. W., and Sodroski, J. (1995). Functional analysis of the vpx, vpr, and nef genes of simian immunodeficiency virus. J. Acquir. Immune Defic. Syndr. Hum. Retrovirol. 8, 335-344.

Persaud, D., Gay, H., Ziemniak, C., Chen, Y. H., Piatak, M. J., Chun, T.-W., et al. (2013). Absence of detectable HIV-1 viremia after treatment cessation in an infant. N. Engl. J. Med. 369, 1828-1835. doi: 10.1056/NEJMoa1302976

Persaud, D., Siberry, G. K., Ahonkhai, A., Kajdas, J., Monie, D., Hutton, N., et al. (2004). Continued production of drug-sensitive human immunodeficiency virus type 1 in children on combination antiretroviral therapy who have undetectable viral loads. J. Virol. 78, 968-979. doi: 10.1128/JVI.78.2.968-979. 2004
Pertel, T., Reinhard, C., and Luban, J. (2011). Vpx rescues HIV-1 transduction of dendritic cells from the antiviral state established by type 1 interferon. Retrovirology 8:49. doi: 10.1186/1742-4690-8-49

Pincus, T., Hartley, J. W., and Rowe, W. P. (1971). A major genetic locus affecting resistance to infection with murine leukemia viruses. J. Exp. Med. 133, 12191233. doi: 10.1084/jem.133.6.1219

Rafati, H., Parra, M., Hakre, S., Moshkin, Y., Verdin, E., and Mahmoudi, T. (2011). Repressive LTR nucleosome positioning by the BAF complex is required for HIV latency. PLoS Biol. 9:e1001206. doi: 10.1371/journal.pbio.1001206

Rasmussen, T. A., Tolstrup, M., Brinkmann, C. R., Olesen, R., Erikstrup, C., Solomon, A., et al. (2014). Panobinostat, a histone deacetylase inhibitor, for latent-virus reactivation in HIV-infected patients on suppressive antiretroviral therapy: a phase 1/2, single group, clinical trial. Lancet HIV 1, e13-e21. doi: 10.1016/S2352-3018(14)70014-1

Re, F., Braaten, D., Franke, E. K., and Luban, J. (1995). Human immunodeficiency virus type $1 \mathrm{Vpr}$ arrests the cell cycle in G2 by inhibiting the activation of p34cdc2-cyclin B. J. Virol. 69, 6859-6864.

Reinhard, C., Bottinelli, D., Kim, B., and Luban, J. (2014). Vpx rescue of HIV1 from the antiviral state in mature dendritic cells is independent of the intracellular deoxynucleotide concentration. Retrovirology 11:12. doi: 10.1186/ 1742-4690-11-12

Rey-Cuillé, M. A., Berthier, J. L., Bomsel-Demontoy, M. C., Chaduc, Y., Montagnier, L., Hovanessian, A. G., et al. (1998). Simian immunodeficiency virus replicates to high levels in sooty mangabeys without inducing disease. J. Virol. 72, 3872-3886.

Riess, M., Fuchs, N. V., Idica, A., Hamdorf, M., Flory, E., Pedersen, I. M., et al. (2017). Interferons induce expression of SAMHD1 in monocytes through down-regulation of miR-181a and miR-30a. J. Biol. Chem. 292, 264-277. doi: 10.1074/jbc.M116.752584

Rivière, L., Gerossier, L., Ducroux, A., Dion, S., Deng, Q., Michel, M.-L., et al. (2015). HBx relieves chromatin-mediated transcriptional repression of hepatitis B viral cccDNA involving SETDB1 histone methyltransferase. J. Hepatol. 63, 1093-1102. doi: 10.1016/j.jhep.2015.06.023

Robbez-Masson, L., Tie, C. H. C., Conde, L., Tunbak, H., Husovsky, C., Tchasovnikarova, I. A., et al. (2018). The HUSH complex cooperates with TRIM28 to repress young retrotransposons and new genes. Genome Res. 28, 836-845. doi: 10.1101/gr.228171.117

Rosa, A., Chande, A., Ziglio, S., De Sanctis, V., Bertorelli, R., Goh, S. L., et al. (2015). HIV-1 Nef promotes infection by excluding SERINC5 from virion incorporation. Nature 526, 212-217. doi: 10.1038/nature15399

Rowe, W. P., Humphrey, J. B., and Lilly, F. (1973). A major genetic locus affecting resistance to infection with murine leukemia viruses. 3. Assignment of the Fv-1 locus to linkage group 8 of the mouse. J. Exp. Med. 137, 850-853. doi: 10.1084/jem.137.3.850

Sáez-Cirión, A., Bacchus, C., Hocqueloux, L., Avettand-Fenoel, V., Girault, I., Lecuroux, C., et al. (2013). Post-treatment HIV-1 controllers with a longterm virological remission after the interruption of early initiated antiretroviral therapy ANRS VISCONTI study. PLoS Pathog. 9:e1003211. doi: 10.1371/ journal.ppat.1003211

Sakuma, R., Mael, A. A., and Ikeda, Y. (2007). Alpha interferon enhances TRIM5 $\alpha$-mediated antiviral activities in human and rhesus monkey cells. J. Virol. 81, 10201-10206. doi: 10.1128/JVI.00419-07

Saleh, S., Vranckx, L., Gijsbers, R., Christ, F., and Debyser, Z. (2017). Insight into HIV-2 latency may disclose strategies for a cure for HIV-1 infection. J. Virus Erad. 3, 7-14.

Sarkis, P. T. N., Ying, S., Xu, R., and Yu, X.-F. (2006). STAT1-independent cell type-specific regulation of antiviral APOBEC3G by IFN-alpha. J. Immunol. 177, 4530-4540. doi: 10.4049/jimmunol.177.7.4530

Sawyer, S. L., Emerman, M., and Malik, H. S. (2004). Ancient adaptive evolution of the primate antiviral DNA-editing enzyme APOBEC3G. PLoS Biol. 2:e275. doi: 10.1371/journal.pbio.0020275

Sawyer, S. L., Wu, L. I., Emerman, M., and Malik, H. S. (2005). Positive selection of primate TRIM5alpha identifies a critical species-specific retroviral restriction domain. Proc. Natl. Acad. Sci. U.S.A. 102, 2832-2837. doi: 10.1073/pnas. 0409853102

Schaller, T., Bauby, H., Hué, S., Malim, M. H., and Goujon, C. (2014). New insights into an X-traordinary viral protein. Front. Microbiol. 5:126. doi: 10.3389/fmicb. 2014.00126 
Schröder, A. R. W., Shinn, P., Chen, H., Berry, C., Ecker, J. R., and Bushman, F. (2002). HIV-1 integration in the human genome favors active genes and local hotspots. Cell 110, 521-529. doi: 10.1016/S0092-8674(02)00864-4

Schwefel, D., Groom, H. C. T., Boucherit, V. C., Christodoulou, E., Walker, P. A., Stoye, J. P., et al. (2014). Structural basis of lentiviral subversion of a cellular protein degradation pathway. Nature 505, 234-238. doi: 10.1038/nature 12815

Shan, L., Deng, K., Shroff, N. S., Durand, C. M., Rabi, S. A., Yang, H.-C., et al. (2012). Stimulation of HIV-1-specific cytolytic T lymphocytes facilitates elimination of latent viral reservoir after virus reactivation. Immunity 36, 491-501. doi: 10.1016/j.immuni.2012.01.014

Sharova, N., Wu, Y., Zhu, X., Stranska, R., Kaushik, R., Sharkey, M., et al. (2008). Primate lentiviral Vpx commandeers DDB1 to counteract a macrophage restriction. PLoS Pathog. 4:e1000057. doi: 10.1371/journal.ppat.1000057

Sharp, P. M., Bailes, E., Stevenson, M., Emerman, M., and Hahn, B. H. (1996). Gene acquisition in HIV and SIV. Nature 383, 586-587. doi: 10.1038/383586a0

Sheehy, A. M., Gaddis, N. C., Choi, J. D., and Malim, M. H. (2002). Isolation of a human gene that inhibits HIV-1 infection and is suppressed by the viral Vif protein. Nature 418, 646-650. doi: 10.1038/nature00939

Sheehy, A. M., Gaddis, N. C., and Malim, M. H. (2003). The antiretroviral enzyme APOBEC3G is degraded by the proteasome in response to HIV-1 Vif. Nat. Med. 9, 1404-1407. doi: 10.1038/nm945

Sheridan, P. L., Mayall, T. P., Verdin, E., and Jones, K. A. (1997). Histone acetyltransferases regulate HIV-1 enhancer activity in vitro. Genes Dev. 11, 3327-3340. doi: 10.1101/gad.11.24.3327

Sherrill-Mix, S., Lewinski, M. K., Famiglietti, M., Bosque, A., Malani, N., Ocwieja, K. E., et al. (2013). HIV latency and integration site placement in five cell-based models. Retrovirology 10:90. doi: 10.1186/1742-4690-10-90

Shun, M.-C., Raghavendra, N. K., Vandegraaff, N., Daigle, J. E., Hughes, S., Kellam, P., et al. (2007). LEDGF/p75 functions downstream from preintegration complex formation to effect gene-specific HIV-1 integration. Genes Dev. 21, 1767-1778. doi: 10.1101/gad.1565107

Siliciano, J. D., Kajdas, J., Finzi, D., Quinn, T. C., Chadwick, K., Margolick, J. B., et al. (2003). Long-term follow-up studies confirm the stability of the latent reservoir for HIV-1 in resting CD4 ${ }^{+}$T cells. Nat. Med. 9, 727-728. doi: 10.1038/ nm880

Silvestri, G., Fedanov, A., Germon, S., Kozyr, N., Kaiser, W. J., Garber, D. A., et al. (2005). Divergent host responses during primary simian immunodeficiency virus SIVsm infection of natural sooty mangabey and nonnatural rhesus macaque hosts. J. Virol. 79, 4043-4054. doi: 10.1128/JVI.79.7.4043-4054.2005

Silvestri, G., Sodora, D. L., Koup, R. A., Paiardini, M., O’Neil, S. P., McClure, H. M., et al. (2003). Nonpathogenic SIV infection of sooty mangabeys is characterized by limited bystander immunopathology despite chronic high-level viremia. Immunity 18, 441-452. doi: 10.1016/S1074-7613(03)00060-8

Søgaard, O. S., Graversen, M. E., Leth, S., Olesen, R., Brinkmann, C. R., Nissen, S. K., et al. (2015). The depsipeptide romidepsin reverses HIV-1 latency in vivo. PLoS Pathog. 11:e1005142. doi: 10.1371/journal.ppat.1005142

Spivak, A. M., Bosque, A., Balch, A. H., Smyth, D., Martins, L., and Planelles, V. (2015). Ex vivo bioactivity and HIV-1 latency reversal by ingenol dibenzoate and panobinostat in resting $\mathrm{CD} 4^{+} \mathrm{T}$ cells from aviremic patients. Antimicrob. Agents Chemother. 59, 5984-5991. doi: 10.1128/AAC.01077-15

Srivastava, S., Swanson, S. K., Manel, N., Florens, L., Washburn, M. P., and Skowronski, J. (2008). Lentiviral Vpx accessory factor targets VprBP/DCAF1 substrate adaptor for cullin 4 E3 ubiquitin ligase to enable macrophage infection. PLoS Pathog. 4:e1000059. doi: 10.1371/journal.ppat. 1000059

Stivahtis, G. L., Soares, M. A., Vodicka, M. A., Hahn, B. H., and Emerman, M. (1997). Conservation and host specificity of Vpr-mediated cell cycle arrest suggest a fundamental role in primate lentivirus evolution and biology. J. Virol. 71, 4331-4338.

Stopak, K. S., Chiu, Y.-L., Kropp, J., Grant, R. M., and Greene, W. C. (2007). Distinct patterns of cytokine regulation of APOBEC3G expression and activity in primary lymphocytes, macrophages, and dendritic cells. J. Biol. Chem. 282, 3539-3546. doi: 10.1074/jbc.M610138200

Strain, M. C., Little, S. J., Daar, E. S., Havlir, D. V., Gunthard, H. F., Lam, R. Y., et al. (2005). Effect of treatment, during primary infection, on establishment and clearance of cellular reservoirs of HIV-1. J. Infect. Dis. 191, 1410-1418. doi: $10.1086 / 428777$
Stremlau, M., Owens, C. M., Perron, M. J., Kiessling, M., Autissier, P., and Sodroski, J. (2004). The cytoplasmic body component TRIM5alpha restricts HIV-1 infection in Old World monkeys. Nature 427, 848-853. doi: 10.1038/ nature 02343

Stremlau, M., Perron, M., Lee, M., Li, Y., Song, B., Javanbakht, H., et al. (2006). Specific recognition and accelerated uncoating of retroviral capsids by the TRIM5alpha restriction factor. Proc. Natl. Acad. Sci. U.S.A. 103, 5514-5519. doi: 10.1073/pnas.0509996103

Sumpter, B., Dunham, R., Gordon, S., Engram, J., Hennessy, M., Kinter, A., et al. (2007). Correlates of preserved $\mathrm{CD} 4^{+} \mathrm{T}$ cell homeostasis during natural, nonpathogenic simian immunodeficiency virus infection of sooty mangabeys: implications for AIDS pathogenesis. J. Immunol. 178, 1680-1691. doi: 10.4049/ jimmunol.178.3.1680

Talbert, P. B., and Henikoff, S. (2006). Spreading of silent chromatin: inaction at a distance. Nat. Rev. Genet. 7, 793-803. doi: 10.1038/nrg1920

Tanaka, Y., Marusawa, H., Seno, H., Matsumoto, Y., Ueda, Y., Kodama, Y., et al. (2006). Anti-viral protein APOBEC3G is induced by interferon-alpha stimulation in human hepatocytes. Biochem. Biophys. Res. Commun. 341, 314319. doi: 10.1016/j.bbrc.2005.12.192

Tareen, S. U., Sawyer, S. L., Malik, H. S., and Emerman, M. (2009). An expanded clade of rodent Trim5 genes. Virology 385, 473-483. doi: 10.1016/j.virol.2008. 12.018

Tartour, K., Appourchaux, R., Gaillard, J., Nguyen, X.-N., Durand, S., Turpin, J., et al. (2014). IFITM proteins are incorporated onto HIV-1 virion particles and negatively imprint their infectivity. Retrovirology 11:103. doi: 10.1186/s12977014-0103-y

Tchasovnikarova, I. A., Timms, R. T., Douse, C. H., Roberts, R. C., Dougan, G., Kingston, R. E., et al. (2017). Hyperactivation of HUSH complex function by Charcot-Marie-Tooth disease mutation in MORC2. Nat. Genet. 49, 1035-1044. doi: $10.1038 /$ ng.3878

Tchasovnikarova, I. A., Timms, R. T., Matheson, N. J., Wals, K., Antrobus, R., Göttgens, B., et al. (2015). GENE SILENCING. Epigenetic silencing by the HUSH complex mediates position-effect variegation in human cells. Science 348, 1481-1485. doi: 10.1126/science.aaa7227

Tréand, C., du Chéné, I., Brès, V., Kiernan, R., Benarous, R., Benkirane, M., et al. (2006). Requirement for SWI/SNF chromatin-remodeling complex in Tat-mediated activation of the HIV-1 promoter. EMBO J. 25, 1690-1699. doi: 10.1038/sj.emboj.7601074

Tristem, M., Purvis, A., and Quicke, D. L. (1998). Complex evolutionary history of primate lentiviral vpr genes. Virology 240, 232-237. doi: 10.1006/viro.1997.8929

Ueno, F., Shiota, H., Miyaura, M., Yoshida, A., Sakurai, A., Tatsuki, J., et al. (2003). Vpx and Vpr proteins of HIV-2 up-regulate the viral infectivity by a distinct mechanism in lymphocytic cells. Microbes Infect. 5, 387-395. doi: 10.1016/S1286-4579(03)00042-X

Usami, Y., Wu, Y., and Göttlinger, H. G. (2015). SERINC3 and SERINC5 restrict HIV-1 infectivity and are counteracted by Nef. Nature 526, 218-223. doi: 10. 1038/nature 15400

Van Damme, N., Goff, D., Katsura, C., Jorgenson, R. L., Mitchell, R., Johnson, M. C., et al. (2008). The interferon-induced protein BST-2 restricts HIV-1 release and is downregulated from the cell surface by the viral Vpu protein. Cell Host Microbe 3, 245-252. doi: 10.1016/j.chom.2008.03.001

Van Lint, C., Emiliani, S., Ott, M., and Verdin, E. (1996). Transcriptional activation and chromatin remodeling of the HIV-1 promoter in response to histone acetylation. EMBO J. 15, 1112-1120. doi: 10.1002/j.1460-2075.1996.tb00449.x

Verdin, E., Paras, P., and Van Lint, C. (1993). Chromatin disruption in the promoter of human immunodeficiency virus type 1 during transcriptional activation. EMBO J. 12, 3249-3259. doi: 10.1002/j.1460-2075.1993.tb05994.x

Vranckx, L. S., Demeulemeester, J., Saleh, S., Boll, A., Vansant, G., Schrijvers, R., et al. (2016). LEDGIN-mediated inhibition of integrase-LEDGF/p75 interaction reduces reactivation of residual latent HIV. EBioMedicine 8, 248-264. doi: 10. 1016/j.ebiom.2016.04.039

Walker-Sperling, V. E., Pohlmeyer, C. W., Tarwater, P. M., and Blankson, J. N. (2016). The effect of latency reversal agents on primary $C D 8^{+} \mathrm{T}$ cells: implications for shock and kill strategies for human immunodeficiency virus eradication. EBioMedicine 8, 217-229. doi: 10.1016/j.ebiom.2016.04.019

Wan, Z., and Chen, X. (2014). Triptolide inhibits human immunodeficiency virus type 1 replication by promoting proteasomal degradation of Tat protein. Retrovirology 11:88. doi: 10.1186/s12977-014-0088-6 
Wen, X., Duus, K. M., Friedrich, T. D., and de Noronha, C. M. C. (2007). The HIV1 protein $\mathrm{Vpr}$ acts to promote G2 cell cycle arrest by engaging a DDB1 and Cullin4A-containing ubiquitin ligase complex using VprBP/DCAF1 as an adaptor. J. Biol. Chem. 282, 27046-27057. doi: 10.1074/jbc.M7039 55200

White, C. H., Beliakova-Bethell, N., Lada, S. M., Breen, M. S., Hurst, T. P., Spina, C. A., et al. (2018). Transcriptional modulation of human endogenous retroviruses in primary $\mathrm{CD}^{+}{ }^{+} \mathrm{T}$ cells following vorinostat treatment. Front. Immunol. 9:603. doi: 10.3389/fimmu.2018.00603

Wiegand, H. L., Doehle, B. P., Bogerd, H. P., and Cullen, B. R. (2004). A second human antiretroviral factor, APOBEC3F, is suppressed by the HIV-1 and HIV-2 Vif proteins. EMBO J. 23, 2451-2458. doi: 10.1038/sj.emboj.7600246

Wu, C.-H., Yamaguchi, Y., Benjamin, L. R., Horvat-Gordon, M., Washinsky, J., Enerly, E., et al. (2003). NELF and DSIF cause promoter proximal pausing on the hsp70 promoter in Drosophila. Genes Dev. 17, 1402-1414. doi: 10.1101/gad. 1091403

Yu, H., Usmani, S. M., Borch, A., Krämer, J., Stürzel, C. M., Khalid, M., et al. (2013). The efficiency of Vpx-mediated SAMHD1 antagonism does not correlate with the potency of viral control in HIV-2-infected individuals. Retrovirology 10:27. doi: 10.1186/1742-4690-10-27

Yu, X., Yu, Y., Liu, B., Luo, K., Kong, W., Mao, P., et al. (2003). Induction of APOBEC3G ubiquitination and degradation by an HIV-1 Vif-Cul5-SCF complex. Science 302, 1056-1060. doi: 10.1126/science.1089591

Yu, X. F., Ito, S., Essex, M., and Lee, T. H. (1988). A naturally immunogenic virion-associated protein specific for HIV-2 and SIV. Nature 335, 262-265. doi: $10.1038 / 335262 \mathrm{a} 0$
Yu, X. F., Yu, Q. C., Essex, M., and Lee, T. H. (1991). The vpx gene of simian immunodeficiency virus facilitates efficient viral replication in fresh lymphocytes and macrophage. J. Virol. 65, 5088-5091.

Yukl, S. A., Boritz, E., Busch, M., Bentsen, C., Chun, T.-W., Douek, D., et al. (2013). Challenges in detecting HIV persistence during potentially curative interventions: a study of the Berlin patient. PLoS Pathog. 9:e1003347. doi: 10.1371/journal.ppat.1003347

Yurkovetskiy, L., Guney, M. H., Kim, K., Goh, S. L., McCauley, S., Dauphin, A., et al. (2018). Primate immunodeficiency virus proteins Vpx and Vpr counteract transcriptional repression of proviruses by the HUSH complex. Nat. Microbiol. 3, 1354-1361. doi: 10.1038/s41564-018-0256-x

Zhang, H., Yang, B., Pomerantz, R. J., Zhang, C., Arunachalam, S. C., and Gao, L. (2003). The cytidine deaminase CEM15 induces hypermutation in newly synthesized HIV-1 DNA. Nature 424, 94-98. doi: 10.1038/nature01707

Conflict of Interest Statement: The authors declare that the research was conducted in the absence of any commercial or financial relationships that could be construed as a potential conflict of interest.

Copyright (c) 2019 Chougui and Margottin-Goguet. This is an open-access article distributed under the terms of the Creative Commons Attribution License (CC BY). The use, distribution or reproduction in other forums is permitted, provided the original author(s) and the copyright owner(s) are credited and that the original publication in this journal is cited, in accordance with accepted academic practice. No use, distribution or reproduction is permitted which does not comply with these terms. 